\title{
Structure and function of Norrin in assembly and activation of a Frizzled 4-Lrp5/6 complex
}

\author{
Jiyuan Ke, ${ }^{1}$ Kaleeckal G. Harikumar, ${ }^{2}$ Clara Erice, ${ }^{1}$ Chen Chen, ${ }^{1}$ Xin Gu, ${ }^{1}$ Liren Wang, ${ }^{1}$

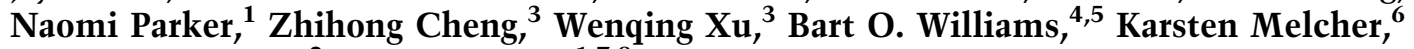 \\ Laurence J. Miller, ${ }^{2}$ and $\mathrm{H}$. Eric $\mathrm{Xu}^{1,7,8}$ \\ ${ }^{1}$ Laboratory of Structural Sciences, Van Andel Research Institute, Grand Rapids, Michigan 49503, USA; ${ }^{2}$ Department of \\ Molecular Pharmacology and Experimental Therapeutics, Mayo Clinic, Scottsdale, Arizona 85259, USA; ${ }^{3}$ Department of \\ Biological Structure, University of Washington School of Medicine, Seattle, Washington 98195, USA; ${ }^{4}$ Center for Skeletal \\ Disease Research, ${ }^{5}$ Laboratory of Cell Signaling and Carcinogenesis, ${ }^{6}$ Laboratory for Structural Biology and Biochemistry, Van \\ Andel Research Institute, Grand Rapids, Michigan 49503, USA; ${ }^{7}$ Van Andel Research Institute/Shanghai Institute of Materia \\ Medica Center, Key Laboratory of Receptor Research, Shanghai Institute of Materia Medica, Chinese Academy of Sciences, \\ Shanghai 201203, China
}

Norrin is a cysteine-rich growth factor that is required for angiogenesis in the eye, ear, brain, and female reproductive organs. It functions as an atypical Wnt ligand by specifically binding to the Frizzled 4 (Fz4) receptor. Here we report the crystal structure of Norrin, which reveals a unique dimeric structure with each monomer adopting a conserved cystine knot fold. Functional studies demonstrate that the novel Norrin dimer interface is required for Fz4 activation. Furthermore, we demonstrate that Norrin contains separate binding sites for Fz4 and for the Wnt ligand coreceptor Lrp5 (low-density lipoprotein-related protein 5) or Lrp6. Instead of inducing Fz4 dimerization, Norrin induces the formation of a ternary complex with $\mathrm{Fz} 4$ and Lrp5/6 by binding to their respective extracellular domains. These results provide crucial insights into the assembly and activation of the Norrin-Fz4-Lrp5/6 signaling complex.

[Keywords: Norrin structure; cystine knot growth factor; Wnt/ $\beta$-catenin signaling; Frizzled 4; low-density lipoprotein receptor-related protein 5/6; tetraspanin 12 ]

Supplemental material is available for this article.

Received August 13, 2013; revised version accepted October 3, 2013.

Wnt signaling plays essential roles in animal embryogenesis, tissue homeostasis, and numerous diseases (Huelsken and Behrens 2002; Logan and Nusse 2004). The canonical Wnt signaling pathway is mediated by the direct binding of Wnts to the Frizzled (Fz) family of receptors and to the Wnt coreceptors Lrp5 (low-density lipoprotein receptor-related protein 5) and Lrp6, which typically results in activation of the $\beta$-catenin/TCF transcriptional pathway (He et al. 2004). Norrin is an atypical Wnt ligand, which can activate $\beta$-catenin signaling through its specific binding to the Fz4 receptor (Xu et al. 2004).

Norrin is encoded by the Norrie disease protein $(N D P)$ gene, which is named after Norrie disease, a rare but severe retinal hypovascularization disease; mutation in the NDP gene also causes the related milder disorder familial exudative vitreoretinopathy (FEVR) (Chen et al. 1993;

${ }^{8}$ Corresponding author

E-mail eric.xu@vai.org.

Article is online at http://www.genesdev.org/cgi/doi/10.1101/gad.228544.113.
Berger 1998). To date, $>100$ different disease-causing NPD mutations have been mapped (Ye et al. 2010). Norrin plays important roles in angiogenesis for not only eye development but also the development of the ear, brain, and female reproductive system (Rehm et al. 2002; Luhmann et al. 2005). Norrin has pronounced neuroprotective properties for retinal neurons (Seitz et al. 2010). Norrin is also required for maintaining the integrity of the blood-brain barrier and blood-retina barrier; loss of the latter is involved in a wide range of diseases, including age-related macular degeneration (AMD) and diabetic macular edema (Wang et al. 2012). Retinal hypovascularization diseases such as diabetic retinopathy, AMD, and retinopathy of prematurity $(\mathrm{ROP})$ are leading causes of vision impairment

(c) $2013 \mathrm{Ke}$ et al. This article is distributed exclusively by Cold Spring Harbor Laboratory Press for the first six months after the full-issue publication date (see http://genesdev.cshlp.org/site/misc/terms.xhtml). After six months, it is available under a Creative Commons License (AttributionNonCommercial 3.0 Unported), as described at http://creativecommons.org/ licenses/by-nc/3.0/. 
and blindness, which collectively affect $\sim 20$ million patients in the United States alone. While Norrie disease and FEVR are rare, Norrin has also been reported to suppress both vascular loss and pathological neovascularization in a mouse model of ROP /Ohlmann et al. 2010), suggesting that recombinant Norrin protein may have therapeutic value for the treatment of not only Norrie disease and FEVR but also ROP, diabetic retinopathy, and AMD.

Like Wnts, Norrin specifically binds with high affinity to the $\mathrm{N}$-terminal extracellular cysteine-rich domain (CRD) of $\mathrm{Fz} 4$ to activate the $\beta$-catenin signaling pathway (Xu et al. 2004). Importantly, mutations in Fz4 cause FEVR, as do mutations in Lrp5 (He et al. 2004), suggesting that Lrp5 may function in the same pathway as Norrin and Fz4. Moreover, cotransfection with Lrp5 (or its close homolog, Lrp6) greatly potentiates Norrin-mediated Fz4 activation (Xu et al. 2004), suggesting that Lrp5/6 may function as Norrin coreceptors, analogous to their role as Wnt coreceptors. However, the mechanism of how Lrp5/6 participate in Norrin/Fz4 signaling remains puzzling because Norrin (in contrast to Wnts) did not show a direct interaction with Lrp5/6 in cell-based binding assays (Xu et al. 2004). A tetraspanin family protein, Tspan 12 , has been identified as an additional factor specific for Norrininduced, but not Wnt-induced, $\beta$-catenin signaling (Junge et al. 2009). Tspan 12 is thought to have chaperone activity to further stabilize Norrin-Fz4 signaling complexes.

Norrin belongs to the cystine knot growth factor superfamily and shows weak homology (sequence identity $\leq 17 \%$ ) with transforming growth factor- $\beta$ (TGF- $\beta$ ) proteins. No structural information is available for Norrin or its close homolog in part due to the difficulties in obtaining pure proteins for structural studies. In this study, we report the expression, purification, crystallization, and structure determination of Norrin. The structure revealed that Norrin forms a unique dimer. Functional studies indicated that the novel Norrin dimer interface is required for Fz4 activation. Structure-based mutagenesis further revealed that Norrin contains separate binding sites for $\mathrm{Fz} 4$ and Lrp5/6. Mutations in either binding site compromise Fz4 activation by Norrin. We demonstrate that Norrin activates Fz4 through assembly of a complex of Norrin, Fz4, Lrp5/6, and Tspan12.

\section{Results}

Active Norrin dimer can be purified as an $M B P$ (maltose-binding protein) fusion protein

Norrin is a small protein containing 11 cysteines and is difficult to purify from mammalian sources (Perez-Vilar and Hill 1997). Previous work that focused on purifying recombinant Norrin protein from insect cells failed to produce Norrin at high purity or in high yield (Shastry and Trese 2003). Similarly, our attempts to express Norrin in mammalian cells were not successful, while expression in standard Escherichia coli systems resulted in the formation of inclusion bodies. We therefore developed a new expression and purification method for Norrin based on an
E. coli system that we used for class B G-protein-coupled receptor (GPCR) extracellular domains (ECDs), which contain several disulfide bonds (see the Materials and Methods; Pioszak and Xu 2008; Pioszak et al. 2008). Using this system, human Norrin (residues 25-133, without its signal peptide) fused to the $C$ terminus of MBP could be expressed as a soluble protein (Fig. 1A).

The purified MBP-Norrin protein formed disulfide bond-linked dimers that migrated at the predicted molecular weight of $106 \mathrm{kDa}$ under nonreducing conditions and $53 \mathrm{kDa}$ under reducing conditions (Fig. 1A). MBPNorrin also runs as a dimer peak on a gel filtration column (Fig. 1B). The fusion protein was active in binding to a mammalian-produced Fz4 CRD fused with an FcH6 tag (Fz4-FcH6), as determined by both biolayer interferometry (Fig. 1C) and AlphaScreen luminescence proximity assays (Fig. 1D). The binding avidity between the MBP-Norrin dimer and Fz4-FcH6 dimer was determined to be $\sim 11 \mathrm{nM}$ by AlphaScreen saturation binding curves (Fig. 1E) and $5 \mathrm{nM}$ by biolayer interferometry (Supplemental Fig. 1A), which is similar to that of mammalian Norrin to Fz4 in an earlier report (Xu et al. 2004). As expected, binding was specific to $\mathrm{Fz} 4$ because we were unable to detect any interaction with the Fz8 CRD (Fig. 1C,D). Recombinant MBP-Norrin was also active in cell-based reporter assays. 293STF cells without Fz4 expression did not respond to exogenously added MBP-Norrin (Fig. 1F). Recombinant MBP-Norrin protein increased the TCF reporter activity of cells transfected with full-length Fz4 by about twofold and of cells cotransfected with Fz4 and Lrp5 to about sixfold in a dose-dependent manner, with an $\mathrm{EC}_{50}$ of $\sim 6 \mathrm{nM}(0.6 \mu \mathrm{g} / \mathrm{mL})$ (Fig. 1F). Cotransfection of 293STF cells with Fz4, Lrp5, and MBP-Norrin mammalian expression vectors caused similar levels of activation, which were about half of those obtained by cotransfection with the untagged Norrin (Fig. 1F,G). This indicates that the purified MBP-Norrin protein is active and selective for activation of $\mathrm{Fz} 4$, consistent with the previous report (Xu et al. 2004). To examine whether Norrin is lipidmodified and therefore hydrophobic, as reported for Wnt ligands (Willert et al. 2003; Takada et al. 2006; Janda et al. 2012), a phase separation experiment was performed. MBP-Norrin expressed from mammalian cells partitioned into the aqueous phase, whereas MBP-rhodopsin (a GPCR membrane protein) partitioned into the detergent phase as expected (Fig. 1H). So, in contrast to Wnts, Norrin is a hydrophilic protein without detectable lipid modification, which is consistent with the fact that Norrin lacks the conserved saposin-like domain of Wnts, where the lipid-modified serine residue is located.

\section{The crystal structure of MBP-Norrin reveals a novel dimeric complex}

We were able to generate high-quality crystals of MBPNorrin that allowed us to determine its structure to $2.4 \AA$ resolution (Table 1). There is excellent electron density for the Norrin molecule in the crystal (Supplemental Fig. 1B). The structure was solved with the phase information derived from the MBP molecular replacement solution, 

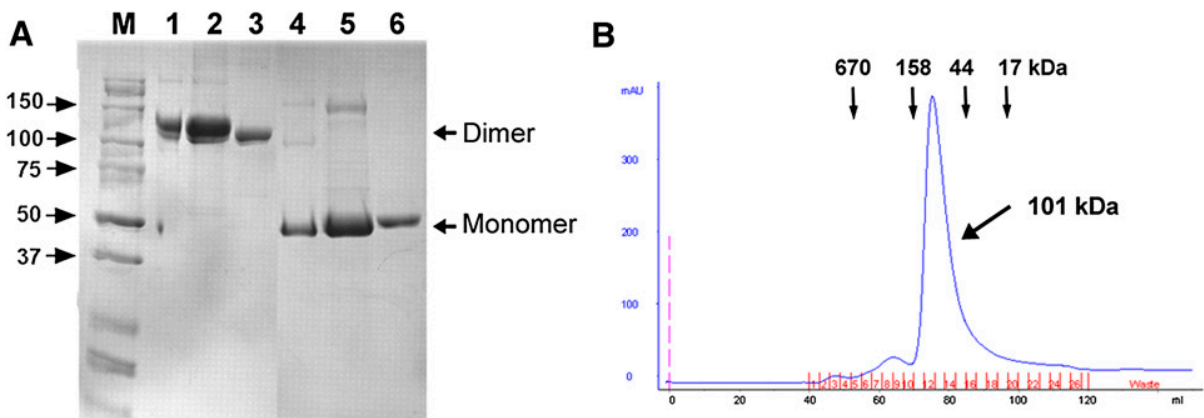

M. Marker, 1\&4. Fz4-Fc, 2\&5. Fz8-Fc, 3\&6. MBP-Norrin,

1-3 (non-reduced), 4-6 (reduced).

C

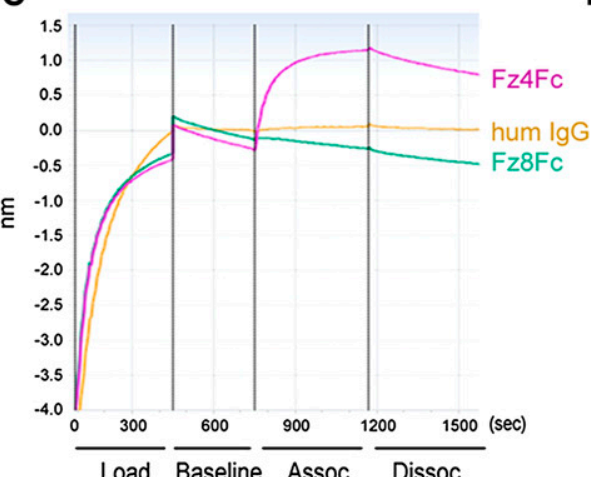

D

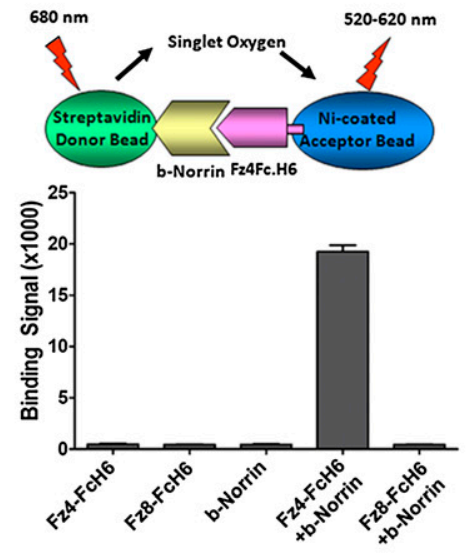

E

$\mathbf{F}$

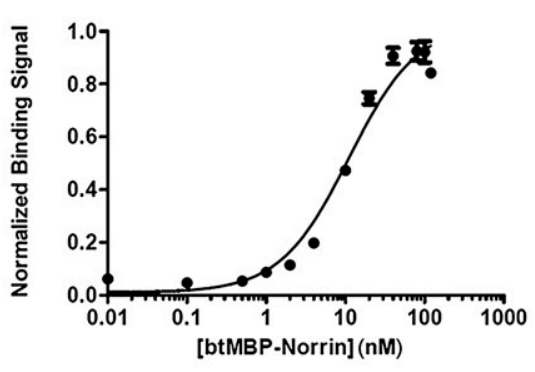

G

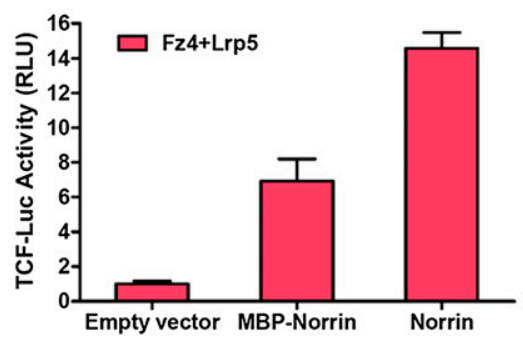

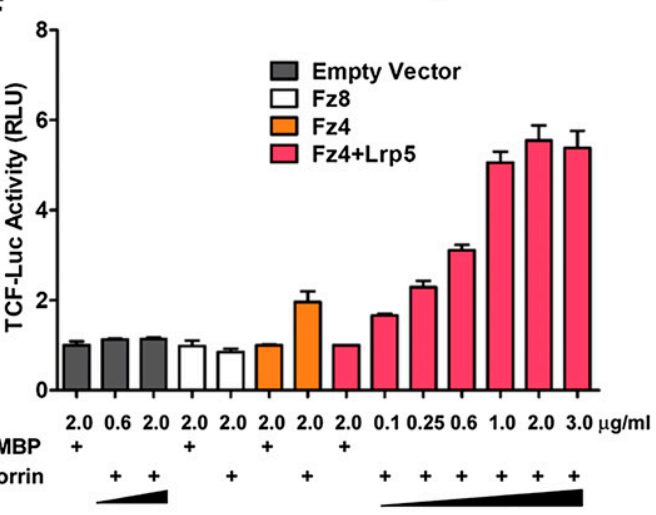

H

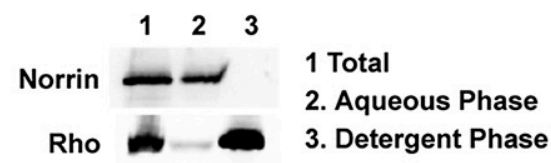

Figure 1. MBP-Norrin protein is functional in binding assays and cell-based reporter assays. $(A)$ SDS-gel analysis of purified Fz4-FcH6, Fz8-FcH6, and MBP-Norrin proteins separated under nonreducing (lanes 1-3) and reducing (lanes 4-6) conditions. Fz4-FcH6 and Fz8FcH6 are fusion proteins whose dimerization is mediated through the disulfide bonds in the Fc portion. MBP-Norrin dimerization is mediated through disulfide bonds in the Norrin molecules. (B) Size exclusion column (120 mL of Superdex) profile for MBP-Norrin. MBP-Norrin eluted at $75 \mathrm{~mL}$, corresponding to the size of a dimer. (C) Binding of MBP-Norrin to Fz4-FcH6, Fz8-FcH6, or human IgG was measured by biolayer interferometry. Protein A biosensors were loaded with Fz4-FcH6, Fz8-FcH6, or human IgG. The loaded biosensors were incubated sequentially with buffer in the baseline phase, MBP-Norrin protein in the association phase, and buffer in the dissociation phase. $(D)$ The binding of $10 \mathrm{nM}$ recombinant biotin-MBP-Norrin protein to $20 \mathrm{nM}$ Fz4-FcH6 or Fz8-FcH6 as measured by AlphaScreen assay $(n=3$; error bars indicate SD). (E) Saturation binding curve for biotin-MBP-Norrin to $4 \mathrm{nM} \mathrm{Fz4-FcH6} \mathrm{as} \mathrm{measured}$ by AlphaScreen assay. The $\mathrm{K}_{\mathrm{d}}$ was determined as $11 \mathrm{nM}$ by nonlinear regression using GraphPad Prism. $(F)$ Bacterially expressed MBPNorrin activates TCF-mediated luciferase reporter activity in Fz4-expressing cells. 293STF cells were transfected with the indicated constructs, and luciferase activity was measured as described. $(G)$ Activation of TCF-mediated luciferase activity in cells coexpressing Fz4 and Lrp5 in the presence or absence of MBP-Norrin or Norrin. $(H)$ The hydrophobicity of MBP-Norrin as determined by phase separation assay. MBP-Rhodopsin was used as a control. The MBP-Norrin samples were probed with an anti-Norrin antibody, and MBPRhodopsin samples were probed with an anti-MBP antibody. 
Table 1. X-ray diffraction data collection and refinement statistics

\begin{tabular}{|c|c|}
\hline & MBP-Norrin \\
\hline \multicolumn{2}{|l|}{ Data collection } \\
\hline APS beamline & 21-ID-G \\
\hline Space group & $\mathrm{P} 2_{1} 2_{1} 2$ \\
\hline Resolution & $50 \AA-2.4 \AA$ \\
\hline Cell parameters & $\begin{array}{c}a=59.5 \AA, b=79.0 \AA \\
c=104.2 \AA ; \alpha=\beta=\gamma=90^{\circ}\end{array}$ \\
\hline Total/unique reflections & $188,712 / 19,865$ \\
\hline Completeness & $100.0 \%(100.0 \%)$ \\
\hline $\mathrm{I} / \sigma$ & $13.1(3.0)$ \\
\hline Redundancy & $9.5(9.0)$ \\
\hline$R_{\mathrm{sym}}$ & $0.156(0.883)$ \\
\hline \multicolumn{2}{|l|}{ Structure determination } \\
\hline Resolution & $50 \AA-2.40 \AA$ \\
\hline Number of reflections & 18,833 \\
\hline Number of residues & 474 \\
\hline Number of solvent molecules & 186 \\
\hline Number of non-H atoms & 3898 \\
\hline$R_{\text {cryst }}$ & $20.0 \%$ \\
\hline$R_{\text {free }}$ & $23.6 \%$ \\
\hline RMSD bonds & $0.011 \AA$ \\
\hline RMSD angles & $1.60^{\circ}$ \\
\hline Average B factor & $41.6 \AA^{2}$ \\
\hline \multicolumn{2}{|l|}{ Ramachandran statistics } \\
\hline Most favored regions & $91.1 \%$ \\
\hline Additional allowed regions & $8.7 \%$ \\
\hline Generously allowed regions & $0.2 \%$ \\
\hline Disallowed regions & $0 \%$ \\
\hline
\end{tabular}

which revealed that MBP is involved in crystal packing interactions (Supplemental Fig. 2A). Thus, the inclusion of MBP as a fusion tag not only helped increase the solubility of Norrin but also aided in crystallization and structure determination of the fused protein.

In the structure, MBP-Norrin is arranged as a homodimer with one monomer per asymmetric unit (Supplemental Fig. 2B). Each Norrin monomer consists solely of $\beta$ strands and loops (no $\alpha$ helices) and forms a very flat structure $(59 \AA \times 16 \AA \times 6 \AA)$ that includes a cystine knot motif (Fig. 2A). The motif contains an unusual clustering of three disulfide bonds, two of which, with their connecting peptide backbone, form a ring structure that is threaded by a third disulfide bond. In the Norrin structure, the cystine knot is near the center of each monomer, with two disulfide bonds (C65-C126 and C69-C128) and four amino acid backbones forming an eight-residue ring through which a third disulfide bond (C39-C96) passes (Fig. 2A; Supplemental Fig. 8B). On the left side of the cystine knot are four $\beta$ strands ( $\beta 1$ to $\beta 4$ ) that form two anti-parallel $\beta$ hairpins. The $\beta$ hairpins are stabilized on one end by the cystine knot structure and on the other side by a Norrin-specific disulfide bond $(\mathrm{C} 55-\mathrm{C} 110)$ that connects the $\beta 1-\beta 2$ loop with the $\beta 3-\beta 4$ loop. On the right side of the cystine knot is an additional $\beta$ hairpin formed by strands $\beta 2^{\prime}$ and $\beta 3^{\prime}$.

The Norrin dimer is formed by two highly intertwined monomers that bury $1543 \AA^{2}$ of surface area (Fig. $2 B$ ). In contrast to only one intermolecular disulfide bond (C95C95) predicted previously (Smallwood et al. 2007), three disulfide bonds were observed between the two monomers: two disulfide bonds (C93-C95 and C95-C93) that link the two adjacent $\beta 3$ ' strands from each monomer and one disulfide bond (C131-C131) that links the $\mathrm{C}$ termini of both monomers (Fig. 2C). The dimeric interface is further stabilized by intermolecular hydrogen bonds from $\beta 2$ ' of one monomer to $\beta 2$ and $\beta 4$ of the other monomer (Fig. 2D). As is typical for cystine knot growth factors, Norrin monomers lack a hydrophobic core on their own but, in the context of dimers, form a well-defined hydrophobic core that is partly mediated by symmetric packing of F89 and I123 from each monomer (Fig. 2E). Its extensive dimer interface suggests that Norrin functions as a dimeric protein.

\section{Structural comparison of Norrin with TGF- $\beta$ growth factors}

The cystine knot structural motif is found in a number of growth factors, including nerve growth factor (NGF), TGF- $\beta$, bone morphogenetic protein, platelet-derived growth factor (PDGF-BB), and glycoprotein hormones such as human chorionic gonadotropin (Sun and Davies 1995). Using TGF- $\beta$ as a typical example, the structure of Norrin was compared with that of TGF- $\beta 3$ (Supplemental Fig. 3). Both monomeric Norrin and monomeric TGF- $\beta 3$ have a highly conserved cystine knot structure but also have significant differences in four regions (Supplemental Fig. 3A). The most noticeable difference is the insertion between $\beta 2$ and $\beta 3$, which forms a two-stranded $\beta$ hairpin with a long loop in Norrin (Supplemental Fig. 3A, box 1) and an $\alpha$-helical structure in TGF- $\beta 3$. A second difference is that the $\beta 1-\beta 2$ and $\beta 3-\beta 4$ loops are linked by a disulfide bond in Norrin (Supplemental Fig. 3A, box 2) but are not constrained in TGF- $\beta 3$. Other differences are the $\mathrm{N}$ terminus and the region between $\beta 1$ and $\beta 2$, which form $\alpha$ helices in TGF- $\beta 3$ (Supplemental Fig. 3A, boxes 3 and 4 ) but form loop structures in Norrin. Norrin has an overall L-shaped structure, whereas that of TGF- $\beta 3$ is more planar. Both Norrin and TGF- $\beta$ proteins form homodimers. TGF$\beta 3$ forms a single disulfide bond-linked dimer, whereas Norrin forms a dimer linked by three disulfide bonds, with the Norrin dimer having a more extended, curved shape due to the unique insertion between $\beta 2$ and $\beta 3$ (Supplemental Fig. 3B). The comparison suggests that the cystine knot structural fold is very plastic and can tolerate many different variations, while dimerization is a common feature to maintain the structural integrity.

\section{The dimeric interface of Norrin is important for its function}

To understand the role of the dimer conformation for Norrin function, we mutated the three intermolecular disulfide bond-forming cysteines either individually (C93A, C95A, or C131A) or in combination (C93A/ C95A/C131A). The mutant and wild-type Norrin constructs were transfected with Fz4 or Fz4 plus Lrp5/6 to assess their ability to activate the $\beta$-catenin/TCF luciferase reporter. Transfection of wild-type Norrin with Fz4, but not with Lrp5 or Lrp6, activated the TCF re- 

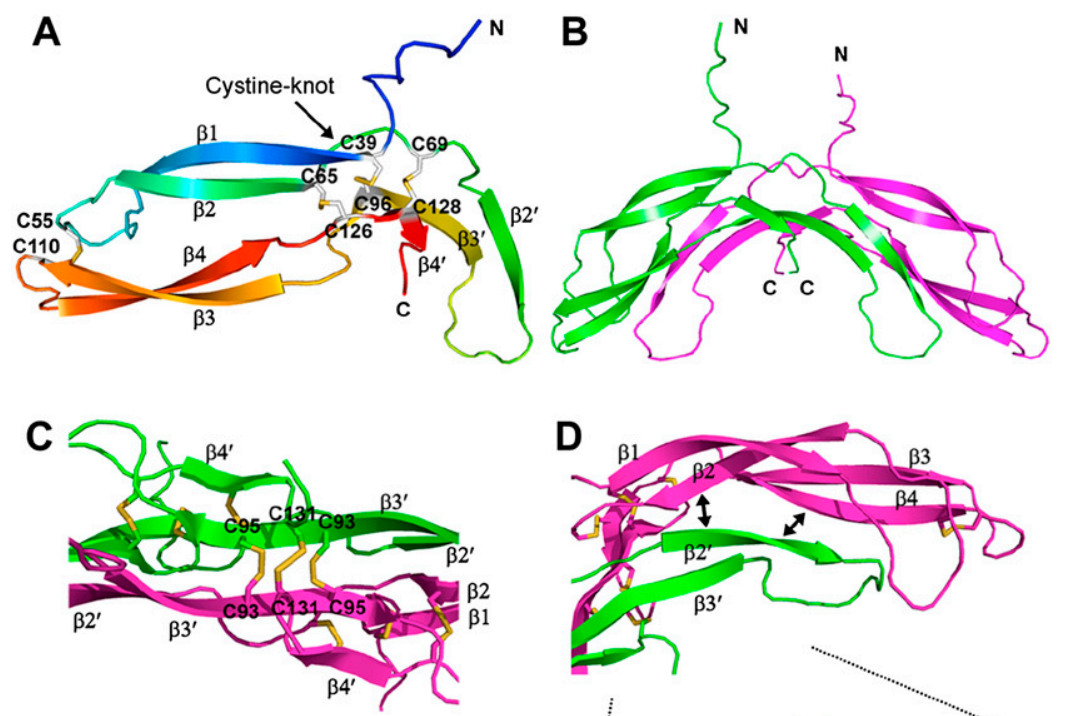

E
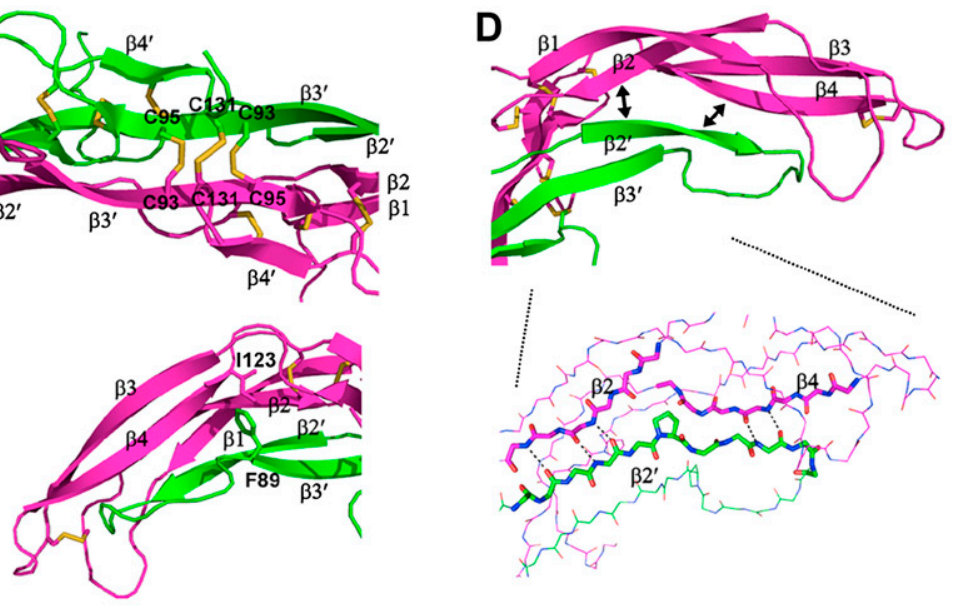

$\mathbf{F}$

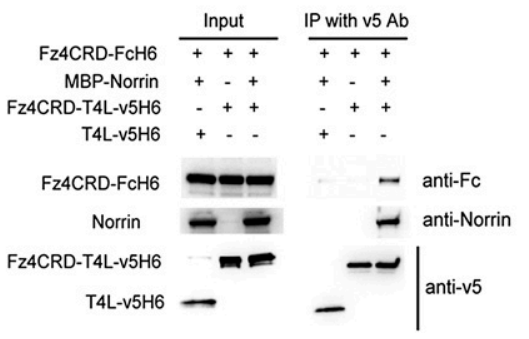

porter, and cotransfection with Fz4 plus Lrp5/Lrp6 further increased reporter activity (Supplemental Fig. 4A). Previous work showed that the C95A mutant of Norrin still forms disulfide bond-linked dimers (Perez-Vilar and Hill 1997). Consistently, single mutation of one of the three intermolecular disulfide bond-forming cysteines resulted in modest reduction of TCF reporter activity in the presence or absence of Lrp5/6 (Supplemental Fig. 4B,C). In contrast, mutation of all three cysteines nearly abolished the reporter activity in the absence of Lrp5/6 and reduced more than half of the reporter activity in the presence of Lrp5/6 (Supplemental Fig. 4B,C). Together, these data support the importance of the intermolecular disulfide bonds for dimer formation and Norrin activation of Fz4. We also mutated F89 and I123, the two key residues that form hydrophobic interactions at the dimeric interface (Fig. 2E), to the hydrophilic residues arginine and asparagine, respectively. The F89R mutant reduced the TCF reporter activity to nearly the same degree as the triple-cysteine mutant; the effect of the I123N mutation (a Norrie disease mutation) was slightly less severe (Supplemental Fig. 4B,C). These data further support the important role of the hydrophobic interface for the integrity of Norrin structure and function.
Figure 2. The crystal structure of human Norrin protein. (A) The structure of the Norrin monomer is shown in a rainbow color scheme from the $\mathrm{N}$ terminus (blue) to the $\mathrm{C}$ terminus (red), with four intramolecular disulfide bonds (C39-C96, C65-C126, C69-C128, and C55C110) shown as stick models. MBP is omitted for clarity. $(B)$ The structure of a Norrin dimer with the monomers colored green and magenta. (C) The Norrin dimer, showing the three intermolecular disulfide bonds (C93-C95, C95-C93, and C131-C131). (D) The Norrin dimer is further stabilized by intermolecular hydrogen bond interactions between $\beta 2^{\prime}$ of one monomer (green) and $\beta 2$ and $\beta 4$ from the other monomer (magenta). (E) Hydrophobic F89-I123 interaction at the dimeric interface. $(F)$ Each Norrin dimer binds to two Fz4 CRDs. Fz4-T4L-v5H6 or T4Lv5H6 was immunoprecipitated with v5-agarose beads. Coprecipitated MBP-Norrin and Fz4-FcH6 were detected by tag-specific antibodies.
Fz4 receptors form stable dimers in the absence of Norrin

Based on the Norrin dimeric structure, we initially favored a model of Norrin activation of $\mathrm{Fz} 4$ through ligand-induced Fz4 receptor dimerization. To determine whether one Norrin dimer can bind two Fz4 CRDs, we incubated MBP-Norrin with two Fz4 CRD proteins, each tagged with either a T4 lysozyme and v5H6 fusion tag (T4L-v5H6) or a FcH6 tag. Fz4 CRD-FcH6 coimmunoprecipitated with Fz4 CRD-T4Lv5H6 in the presence, but not the absence, of MBP-Norrin, demonstrating that each Norrin dimer indeed binds to two Fz4 CRDs (Fig. 2F). We next used bioluminescence resonance energy transfer (BRET) (Harikumar et al. 2007) to examine the effect of Norrin on Fz4 fulllength receptor dimerization using $\mathrm{Fz} 4$ receptors tagged with yellow fluorescent protein (YFP) or Renilla luciferase (Rlu). As controls, we observed Fz4 surface expression when cells were transfected with either YFP- or Rlutagged Fz4 (Supplemental Fig. 5A). To our surprise, fulllength Fz4 receptors strongly interacted with each other in the absence of Norrin, producing a BRET signal as strong as that of the positive control, a covalent Rlu-YFP fusion 
protein (Fig. 3A). To further distinguish genuine receptor interaction from random collision, we performed saturation BRET (Harikumar et al. 2007). Coexpression of a constant amount of Fz4-Rlu with increasing amounts of Fz4-YFP generated a hyperbolic, plateau-reaching signal, indicative of a true interaction, whereas titrating Fz4-Rlu with CCK2R-YFP, an unrelated seven-transmembrane (TM) receptor, generated a nonsaturating, quasilinear signal, indicative of random collisions (Fig. 3B). These data suggest that the $\mathrm{Fz} 4$ receptor exists as a dimer in the absence of exogenous ligands. Interestingly, the human protein smoothened, a remote homolog of Fz4, also homodimerizes through its TM domain (Wang et al. 2013).

We then examined the effect of recombinant MBPNorrin on Fz4 dimerization and found that Norrin did not further increase the Fz4 BRET signal; on the contrary, it slightly but reproducibly reduced the signal (Fig. 3C). Cotransfection of Norrin with YFP- and Rlu-tagged Fz4 expression constructs resulted in a similar small signal decrease (Fig. 3C). We did not observe this effect for Norrin R41E, which does not bind to Fz4 (Smallwood et al. 2007), indicating that the BRET decrease depends on Norrin-Fz4 binding (Fig. 3C). Since BRET signals are exquisitely sensitive to small changes in donor-receptor distance and relative donor and acceptor orientations, the signal reduction in the presence of Norrin could indicate that Norrin induces a slight increase in the distance between the Fz4 receptor monomers or a conformational change of Fz4 receptors but not dissociation of the Fz4 dimer. In control experiments, Norrin did not change either the total expression or the surface expression of Fz4 (Supplemental Fig. 5B,C). Together, these data suggest that Fz4 exists as dimers in the absence of Norrin and that Norrin binding does not increase or disrupt Fz4 dimer formation.

To examine whether Fz4 exists as higher-order oligomers in the presence or absence of Norrin, we used a split-
A
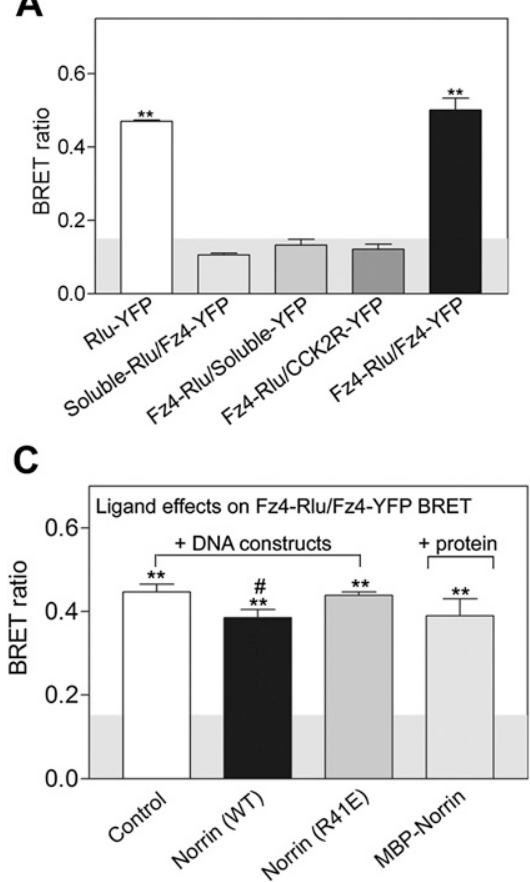

E

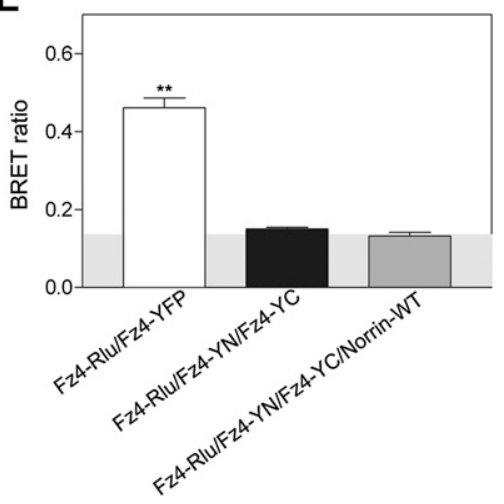

B

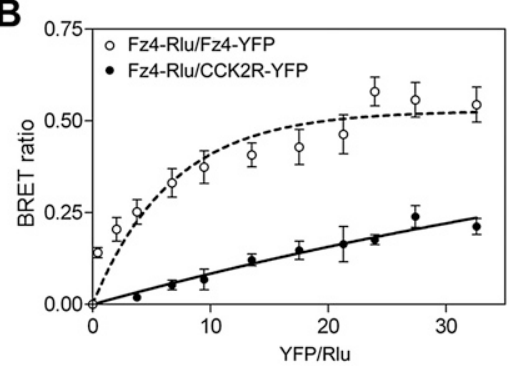

D
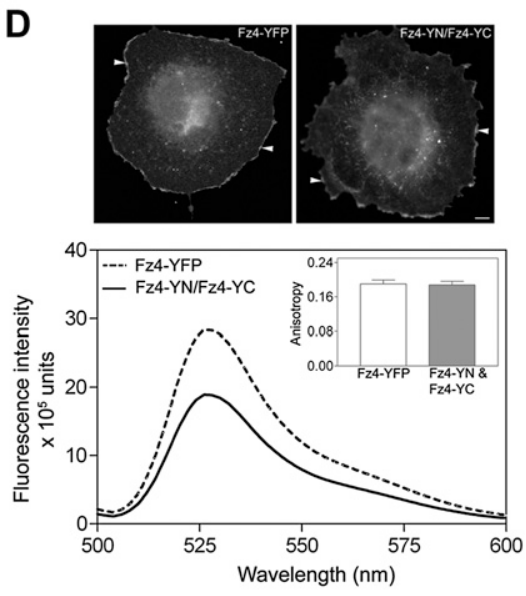

F

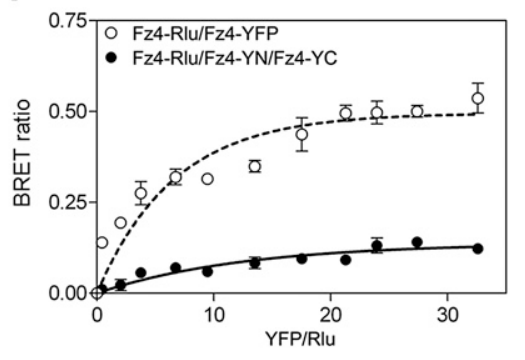

Figure 3. Fz4 receptors dimerize in the absence of Norrin ligand. Shown are the static BRET signals $(A)$ and saturation BRET signals $(B)$ in COS-1 cells expressing both Rlu- and YFP-tagged Fz4 receptors. For $A$, coexpression of soluble Rlu, soluble YFP, or a structurally unrelated type 2 cholecystokinin receptor (CCK2R) was used as negative controls, and the soluble Rlu-YFP was used as a positive control. The shaded area represents the background signal. $(C)$ The effect of the Norrin ligand on the Fz4 BRET signal by coexpression of wild-type and R41E Norrin constructs and vector control or treatment with MBPNorrin protein. Plots represent mean \pm SEM of data from six independent experiments performed in triplicate. $\left(^{\star \star}\right) P<0.01$; $(\#) P<$ 0.05. (D) Bimolecular complementation experiment showing that Fz4 fused to the nonfluorescent complementary halves of YFP and Fz4-YFP-N and Fz4-YFP-C dimerize and establish a strong YFP fluorescent signal on the cell membrane (arrowheads). Bar, $25 \mu \mathrm{M}$. The emission spectrum and anisotropy of this signal were appropriate. (E) Norrin did not induce higher oligomerization of $\mathrm{Fz} 4$ receptors detected by three-component BRET assay. The absence of significant BRET from the Rlu-tagged $\mathrm{Fz} 4$ coexpressed with $\mathrm{Fz} 4$ tagged with YFP-N and YFP-C in the absence or presence of Norrin coexpression. The shaded area represents the background signal as determined using Rlu-tagged Fz4 with CCK2RYFP. A significant static BRET signal between Rlu- and YFP-tagged Fz4 constructs was used as a positive control. $(F)$ The saturation BRET signals for these complexes, establishing the significance of the signal from the Fz4-Rlu/ Fz4-YFP pair and the lack of significance of the signal from coexpression of the Rlutagged Fz4 along with Fz4 tagged with YFP-N and YFP-C. Plots represent mean \pm SEM of data from four independent experiments performed in triplicate. $\left(^{\star \star}\right) P<0.01$. 
YFP BRET assay (Harikumar et al. 2008a) that contained three components: one Fz4 receptor tagged with Rlu (Fz4$\mathrm{Rlu})$, one Fz4 receptor tagged with an N-terminal fragment of YFP (Fz4-YFP-N), and one Fz4 receptor tagged with a C-terminal fragment of YFP (Fz4-YFP-C). Neither of the two YFP fragments fluoresces on their own, but the two fragments form a functional YFP when brought into close proximity. As controls, we observed Fz4 surface expression when cells were transfected with either YFP$\mathrm{N}$-tagged or YFP-C-tagged Fz4 (Supplemental Fig. 5A) and observed YFP fluorescence when cells were transfected with both YFP-N-tagged and YFP-C-tagged Fz4 (Fig. 3D; Supplemental Fig. 5D), confirming the BRET results for constitutive $\mathrm{Fz} 4$ dimer formation. However, regardless of the presence or absence of Norrin, the three-component BRET assay showed no BRET signal above background (Fig. 3E). Similarly, Fz4-Rlu, Fz4-YFP-N, and Fz4-YFP-C did not produce a clear BRET signal in the saturation BRET assay (Fig. 3F). Together, our data suggest that (1) Fz4 exists as constitutive dimers regardless of the presence of Norrin, which is consistent with a previous report (Kaykas et al. 2004); (2) Fz4 dimerization itself is not sufficient for receptor activation; and (3) Norrin does not activate the $\mathrm{Fz} 4$ receptor by inducing Fz4 dimerization. Based on our three-component BRET experiments, Fz4 likely does not form higher-order oligomers. However, we cannot exclude the possibility that $\mathrm{Fz} 4$ can form transient oligomers or oligomers having special geometry that cannot be detected by the BRET assay.

\section{Norrin binds to $\beta$-propeller 1 (BP1) and BP2 of Lrp6}

From genetic studies, Lrp5 is known to be functionally involved in the Norrin/Fz4 signaling complex /Gong et al. 2001), yet previous work has failed to identify a direct interaction between Lrp5/Lrp6 and Norrin fused to alkaline phosphatase (Xu et al. 2004). Thus, how Lrp5 is involved in Norrin/Fz4-mediated $\beta$-catenin signaling remains unclear. We used purified proteins to examine direct interactions between the MBP-Norrin and the Lrp6 ECD using the highly sensitive AlphaScreen assay. The Lrp6 ECD contains four $\beta$-propeller domains (BP1-4). Biotinylated MBP-Norrin interacted with Lrp6 $\beta$-propeller domains 1-4 (BP1-4) and BP1-2 but not BP3-4 (Fig. 4A, lanes 5,9). The interaction between biotinylated MBP-Norrin and Lrp6 BP1-2 can be competed with excess unlabeled MBPNorrin, but not with MBP, with an $\mathrm{IC}_{50}$ of $\sim 570 \mathrm{nM}$ and $\mathrm{K}_{\mathrm{d}}$ of $\sim 450 \mathrm{nM}$ using a homologous competition assay (Fig. 4B,C).

Because adding Fz4 CRD protein did not interfere with the interaction between MBP-Norrin and Lrp6 BP1-2 (Fig. 4A, lane 9 vs. 10) and Lrp6 BP3-4 did not inhibit the interaction between MBP-Norrin and Fz4-FcH6 (Fig. 4A, lane 16 vs. 17), we conclude that Lrp6 BP1-2 and Fz4 CRD bind to different regions of Norrin.

The peptide motif Asn-Ala-Ile-Lys within DKK1 and several other Wnt inhibitors specifically binds to the Lrp6 BP1 domain (Bourhis et al. 2011). We confirmed the interaction between biotinylated DKK1 peptide and His8tagged Lrp6 BP1-2 by AlphaScreen assay (Supplemental

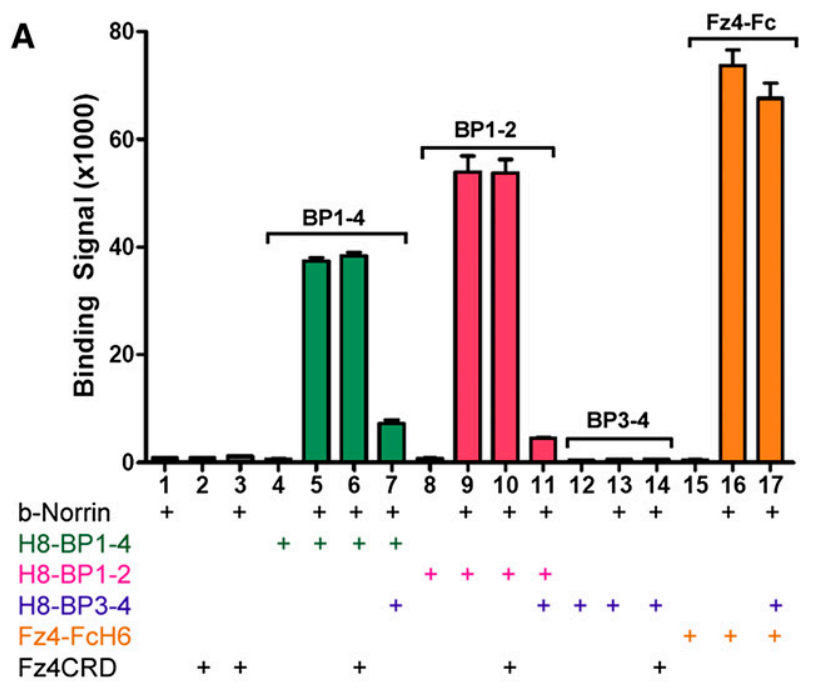

B

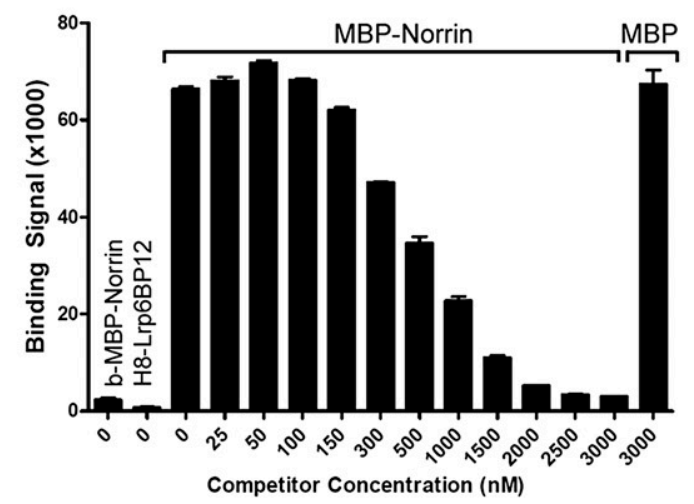

C

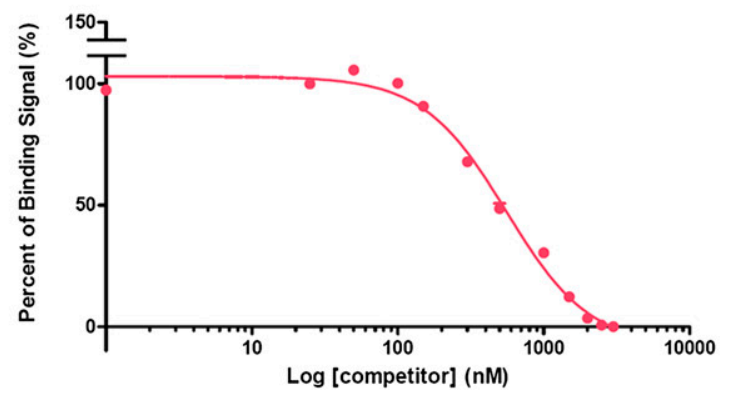

Figure 4. Norrin specifically interacts with Lrp6 BP1-2. (A) AlphaScreen interactions between $30 \mathrm{nM}$ purified biotinMBP-Norrin and $30 \mathrm{nM}$ indicated proteins. (B) The binding affinity for the interaction between MBP-Norrin and Lrp6 BP12 was determined by a homologous AlphaScreen competition assay using $12 \mathrm{nM}$ Lrp6 BP12 and $120 \mathrm{nM}$ biotinylated MBP-Norrin. The left two bars are negative controls with only single proteins. The last bar shows the signal of the reaction with $\mathrm{MBP}$ as a negative competitor control. $(C)$ The competition data were normalized, and the $\mathrm{IC}_{50}$ was determined as $566 \mathrm{nM}$ by nonlinear regression using GraphPad Prism, which corresponds to a $\mathrm{K}_{\mathrm{d}}$ of $\sim 450 \mathrm{nM}$ by Cheng and Prussoff conversion (Cheng and Prusoff 1973).

Fig. 6A, lane 3) and found that MBP-Norrin competed this interaction with an $\mathrm{IC}_{50}$ of $72 \mathrm{nM}$ (Supplemental Fig. 6A; Supplemental Table 1). This suggests that MBP-Norrin binds to a site on Lrp6 BP1 that overlaps with the binding 
site of Wnt inhibitors. To test whether Norrin can also bind to BP2, we purified BP2 as an Fc fusion protein and measured its binding to MBP-Norrin by biolayer interferometry. Protein A sensors specifically bind to the Fc region of human IgG. Lrp6 BP2-FcH6 bound to a protein A sensor interacted with MBP-Norrin with moderate affinity, whereas a human IgG-bound protein A sensor failed to interact (Supplemental Fig. 6B). The binding between biotinylated MBP-Norrin and BP2-FcH6 was also confirmed by AlphaScreen assay (Supplemental Fig. 6C). Although we were unable to purify isolated BP1 as a stable protein, the BP2 binding data and the Norrin competition of DKK1 peptide binding to BP1 suggest that the Norrin dimer is capable of binding to both the BP1 and BP2 domains of Lrp6.

Norrin contains separate binding sites for Lrp6 BP1-2 and the Fz4 CRD

If Norrin has separate binding sites for Lrp5/6 and Fz4, then two classes of Norrin mutations should exist that selectively disrupt those interactions. Indeed, two classes of Norrin mutations have been identified by systematic mutagenesis of Norrin (Smallwood et al. 2007). One class of mutations, including R41E, H43A/Y44A/V45A, M59A/ L60A/L61A, and Y120A/R121A/Y122A, disrupted Norrin binding to the Fz4 CRD and strongly reduced the ability of Norrin to activate the TCF reporter. Mapping these mutations onto the Norrin structure identified a continuous surface area consisting of the R41, H43, V45, L61, Y120, and Y122 residues (Fig. 5A). This surface is exposed and is not at the dimer interface and therefore is likely the major binding site for Fz4. The other class of mutations, including L52A/Y53A/K54A, K54E, R107E, R109E, and R115E, did not affect Fz4 binding yet reduced the ability of Norrin to activate the TCF reporter. These residues are clustered on the edge of the Norrin molecule in the $\beta 1-\beta 2$ and $\beta 3-\beta 4$ loop regions that are linked through the C55C110 disulfide bond (Fig. 5A). Interestingly, the C55A/ C110A mutation, which did not affect Fz4 binding, also reduced the ability of Norrin to activate the TCF reporter (Smallwood et al. 2007). We hypothesize that these residues reduce Norrin signaling activity by reducing or disrupting Norrin binding to Lrp6.

To test our hypotheses, we engineered a number of Norrin mutations and tested their abilities to activate the TCF reporter in the presence of Fz4 and Lrp6. Mutations that interfered with Fz4 binding (R41E, H43A/V45A, L61A, and Y120A/Y122A) or putative Lrp5/6 binding (K54E, R107E, R109E, K54E/R109E, and C55A/C110A) reduced Norrin's signaling activity (Fig. 5B). We then purified three Norrin mutant proteins (R41E, K54E/ $\mathrm{R} 109 \mathrm{E}$, and C55A/C110A) to test their binding to Fz4 and Lrp6, respectively. The unlabeled wild-type MBP-Norrin as well as the C55A/C110A and K54E/R109E mutant proteins all efficiently competed the Fz4-binding signal with $\mathrm{IC}_{50}$ values of $34 \mathrm{nM}, 37 \mathrm{nM}$, and $18 \mathrm{nM}$, respectively (Supplemental Table 1). In contrast, the R41E mutant was unable to compete the Fz4-binding signal, indicating that it is severely compromised in Fz4 binding (Fig. 5C).
We then tested the ability of these three mutant proteins to bind Lrp6 BP1-2 by quantitating their competition for the interaction between His8-tagged Lrp6 BP1-2 and the biotin-DKK1 peptide. Wild-type MBP-Norrin competed the interaction with an $\mathrm{IC}_{50}$ of $72 \mathrm{nM}$, while the C55A/C110A and R41E mutants competed with an $\mathrm{IC}_{50}$ of $207 \mathrm{nM}$ and $288 \mathrm{nM}$, respectively (Supplemental Table 1), indicating that both mutants are partially compromised in binding to Lrp6 BP1-2. In contrast, the K54E/ $\mathrm{R} 109 \mathrm{E}$ double mutant was not able to compete the binding between the DKK1 peptide and Lrp6 BP1-2, indicating its inability to bind to Lrp6 (Fig. 5D). Together, these data support our hypothesis that Norrin contains two separate binding sites: One site, in part comprised of residue R41, is for binding of Fz4; the other site, comprised of residues $\mathrm{K} 54, \mathrm{C} 55, \mathrm{R} 109$, and C110, is for the binding of Lrp6. When mapped on the structure, these two binding sites appear to be adjacent but not overlapping (Fig. 5A).

\section{Norrin, Lrp6 BP1-2, and the Fz4 CRD form a ternary complex}

The two binding sites on Norrin for Lrp6 and Fz4 suggest that they may form a ternary complex. To test complex formation, we expressed Fz4-FcH6, MBP-Norrin, and Lrp5NT (which contains the ECD and TM domain of Lrp5 fused to a v5H6 tandem tag). When Lrp5NT protein was immunoprecipitated with v5 antibody, Fz4-FcH6 coimmunoprecipitated in the presence, but not the absence, of MBP-Norrin (Fig. 6A), supporting formation of a ternary Lrp5-Norrin-Fz4 complex in which MBP-Norrin interacts with both the Lrp5 ECD and the Fz4 CRD. We confirmed these results with a three-hybrid biolayer interferometry assay. When Fz4-FcH6-bound biosensors were incubated first with MBP-Norrin and then with Lrp6 BP1-2, Lrp6 BP1-2 was able to further bind to MBPNorrin prebound to Fz4-FcH6 (Supplemental Fig. 7A). Fz4-bound MBP-Norrin also interacted weakly with Lpr6 BP1-4 but not BP3-4. As controls, the Fz4 CRD did not significantly interact with any of the Lrp6 ECD fragments (Supplemental Fig. 7B), while MBP-Norrin could interact with both the Fz4 CRD (Fig. 1C,D) and the Lrp6 ECD (Fig. 4A). These data indicate that Norrin can bind simultaneously to the Fz4 CRD and Lrp6 BP1-2 to induce the formation of a ternary complex.

\section{The roles of Lrp5/6 and Tspan12 in Norrin-mediated $\beta$-catenin signaling}

To examine the functional importance of Lrp5 for Norrin signaling, we tested whether Lrp5-NT, which lacks the intracellular domain (Fig. 6B), could interfere with Norrinmediated signaling. Previous studies showed that Lrp5-NT acts as a dominant-negative inhibitor of the Wnt/ $\beta$-catenin signaling pathway (Tamai et al. 2000) presumably because Lrp5-NT is insufficient for signaling but competes with endogenous Lrp5/6 for Wnt ligand binding. As positive controls, Norrin activated the TCF reporter in 293STF cells transfected with Fz4, and cotransfection with Lrp5 further enhanced the reporter activity (Fig. 6B). Due to 


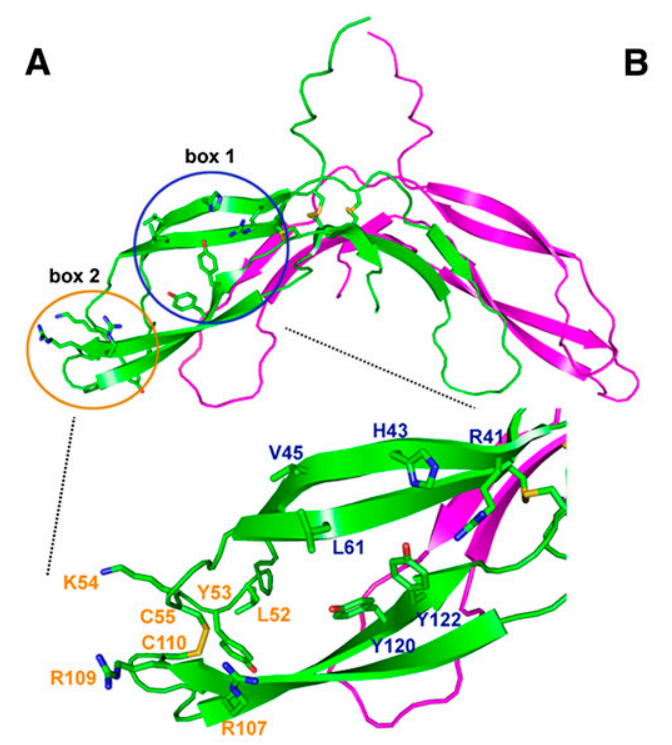

B
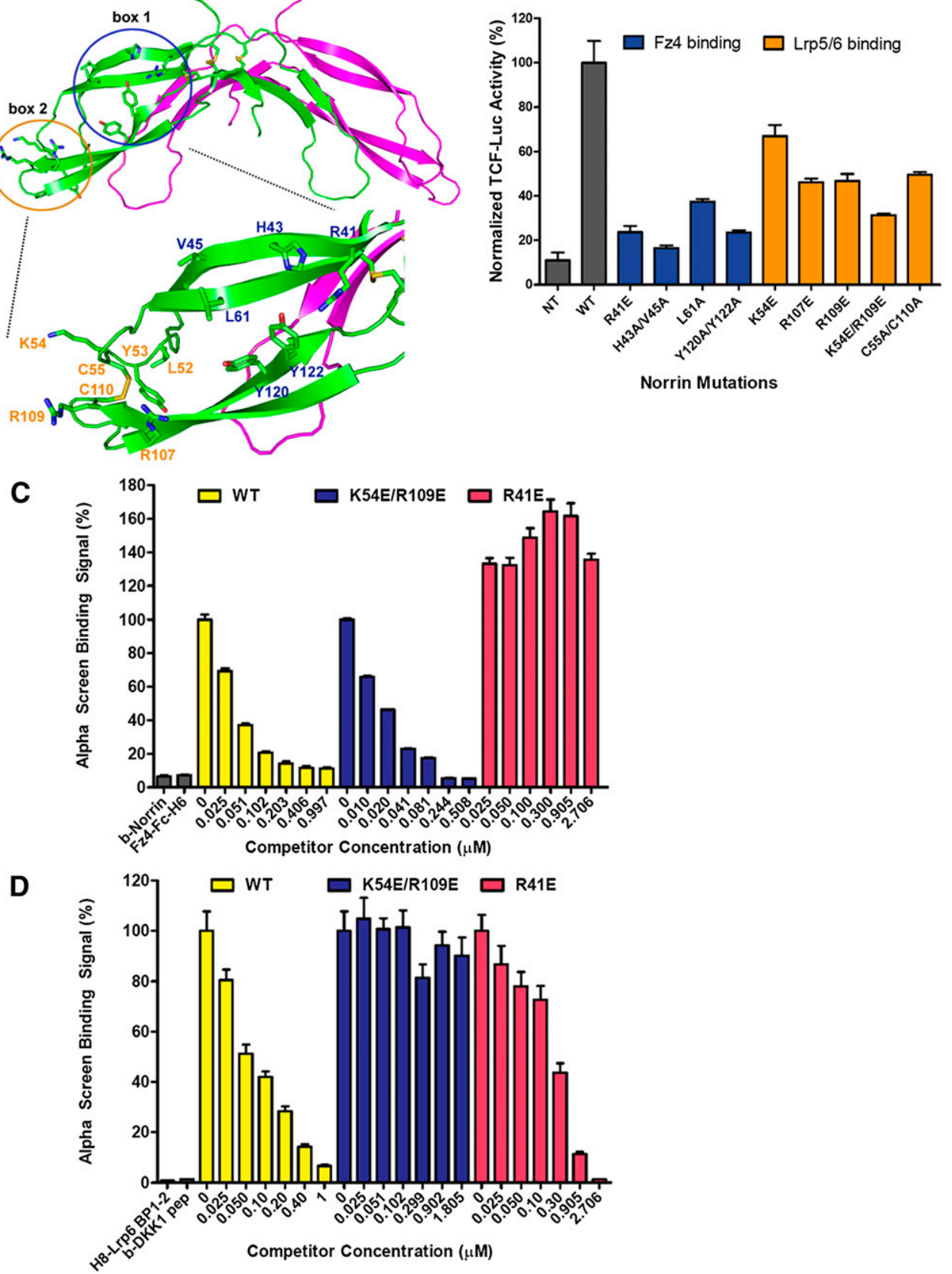

Figure 5. Norrin contains separate binding sites for Fz4 and Lrp5/6. (A) A ribbon diagram of the Norrin dimer with the putative residues involved in binding to Fz4 (blue) or Lrp5/6 (orange) shown as stick models. (B) The effect of mutations of the above surface residues on Norrin function. 293STF cells were transfected with Fz4+Lrp6 in the presence or absence of Norrin wild type (WT) or mutants $(n=3$; error bars indicate SD). (C) AlphaScreen competition assay using MBP-Norrin wild-type or mutant proteins to compete the interaction between biotin-MBP-Norrin and Fz4-FcH6 protein. (D) AlphaScreen competition assay using MBP-Norrin wild-type or

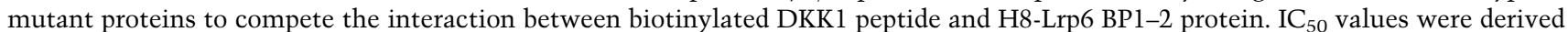
from curve fitting and are shown in Supplemental Table 1.

ubiquitous expression of endogenous Lrp5 and Lrp6, these cotransfection experiments cannot distinguish between either a stimulatory or an essential function of Lrp5 in Norrin/Fz4-mediated signaling. However, the complete loss of responsiveness to Norrin in cells expressing Fz4, endogenous Lrp5/6, and high levels of Lrp5-NT (Fig. 6B) suggests that the Lrp5-N domain competes for a functionally critical interaction between Norrin and endogenous Lrp5/6 and that Lrp5/6 is essential for Norrinmediated Wnt $/ \beta$-catenin signaling. 
Ke et al.

A

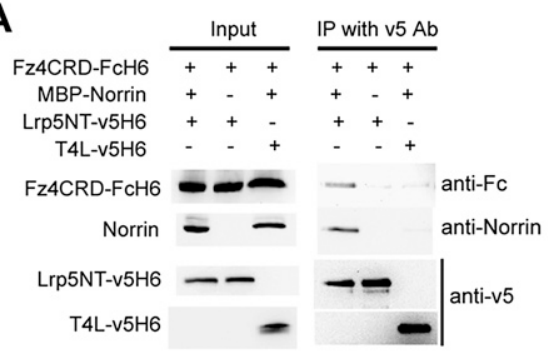

B

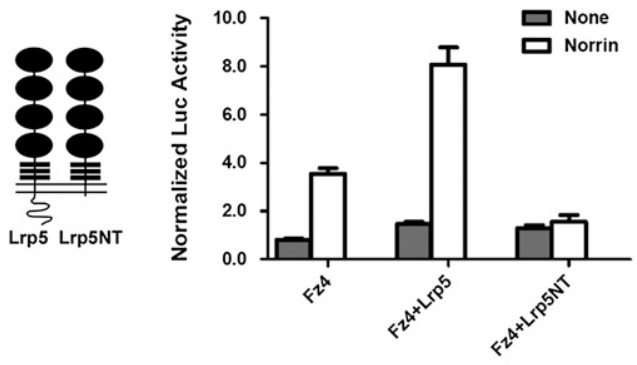

C

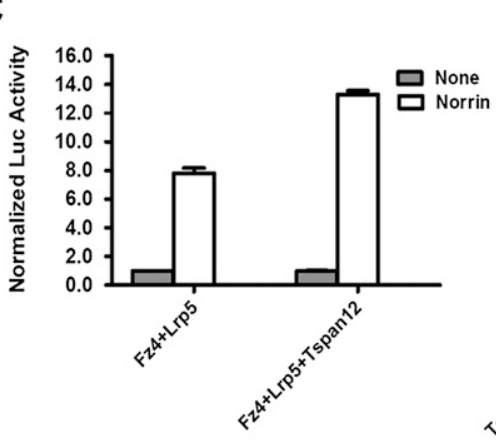

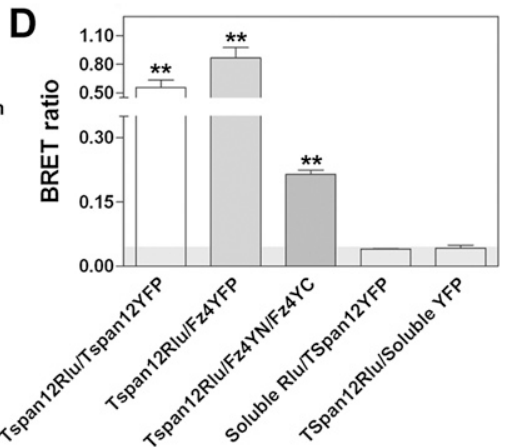

Figure 6. Norrin interacts with Fz4 and Lrp5 to activate downstream $\beta$-catenin signaling, and Tspan12 enhances Norrin/Fz4/Lrp5 signaling by interacting with Fz4. (A) Norrin forms a ternary complex with the Fz4 CRD and the Lrp5 ECD. Lrp5NT-v5H6 and T4L-v5H6 (negative control) were immunoprecipitated with v5-agarose beads. Coimmunoprecipitated MBP-Norrin and Fz4-FcH6 were detected by tag-specific antibodies. $(B$, left $) \mathrm{A}$ diagram of full-length Lrp5 and the Lrp5 C-terminal truncation (Lrp5NT). (Right) Lrp5-NT inhibits Norrin-mediated TCF luciferase reporter activity in a dominant-negative manner. 293STF cells were transfected with Fz4, Fz4+Lrp5, or Fz4+Lrp5-NT in the presence or absence of Norrin expression vector. Luciferase activity was measured as described $(n=3$; error bars indicate SD). (C) Tspan12 increased Norrin/Fz4/Lrp5-mediated $\beta$-catenin signaling. 293STF cells were transfected with Fz4+Lrp5 or Fz4+Lrp5+Tspan12 in the presence or absence of Norrin expression vector. Luciferase activity was measured as described. $(n=3$; error bars indicate SD). (D) Tspan12 strongly interacts with Fz4 receptor on the cell membrane. Shown are the static BRET signals in COS-1 cells expressing Rlu-tagged or YFP-tagged Tspan12 and YFP-tagged, YFP-Ntagged, and YFP-C-tagged Fz4 receptors. The shaded area represents the background signal as determined by coexpression of the Rlu-tagged Tspan 12 with soluble YFP. Plots represent mean \pm SEM of data from three independent experiments performed in triplicate. $\left(^{\star \star}\right) P<0.01$.
Tspan 12 is an additional factor that is specifically involved in Norrin/Fz4/Lrp5 signaling (Junge et al. 2009). Using cell-based assays, we confirmed that cotransfection with Tspan 12 further increased Norrin/Fz4/Lrp5-mediated TCF reporter activity (Fig. 6C). Using the BRET assay, we observed very strong BRET signals from Tspan12-RluTspan12-YFP and Tspan12-Rlu-Fz4-YFP pairs (Fig. 6D), indicating that Tspan 12 can both homodimerize and heterodimerize with Fz4. Interestingly, we observed that Tspan12-Rlu can form a ternary complex with Fz4-YFP-N and Fz4-YFP-C in a split-YFP BRET assay (Fig. 6D). This suggests that Tspan 12 and Fz4 interactions do not disrupt the Fz4-Fz4 dimer interactions. Our experiments are consistent with previous experiments showing that Tspan 12 interacts specifically with Fz4 to facilitate Norrinmediated $\beta$-catenin signaling (Junge et al. 2009).

\section{Discussion}

The expression and purification of Norrin has been a major obstacle that has hampered its structural studies. In this report, we overcame this obstacle by developing a new method for expression and purification of Norrin, which eventually led us to solve its crystal structure. Norrin has a novel dimeric structure, and we were able to map two separate sites on the surface of Norrin, one for binding to $\mathrm{Fz} 4$ receptor and one for binding to Lrp5/6 coreceptor, which helped us establish a model of an activation complex consisting of Norrin, Fz4, Lrp5/6, and Tspan12. These results provide important insights into molecular mechanisms of Norrin signaling and unify the roles of Lrp5/6 as coreceptors in both Wnt and Norrin signaling.

\section{Norrin dimeric structure}

The overall structure feature of Norrin is a homodimer linked through three intermolecular disulfide bonds. This structure is very different from the recent structure of Wnt8 in complex with the Fz8 CRD, which has a monomeric structure with a central "palm" domain and an extended "thumb" and "index finger" (Janda et al. 2012). Importantly, Norrin lacks any lipid modification, which is present in all Wnt proteins and is required for their function (Nusse 2003; Takada et al. 2006). This suggests that the binding between Norrin and Fz4 receptor is not driven by lipid-mediated hydrophobic interactions but through specific protein-protein interactions.

Norrin belongs to the cystine knot growth factor superfamily, and dimer formation is a common theme for these growth factors (Sun and Davies 1995). Consistent with a stable dimer structure, Norrin has extensive interactions 
at the dimer interface, including disulfide bonds, hydrogen bonds, and hydrophobic interactions (Fig. 2C-E). Although the monomeric structure of Norrin contains the conserved cystine knot motif, the dimeric structure of Norrin is different from that of other cystine knot proteins. For instance, NGF forms a dimer in a head-to-head orientation, whereas TGF- $\beta 3$ and PDGF-BB form a dimer in a head-to-tail orientation. NGF, TGF- $\beta 3$, and PDGF-BB all form homodimers through zero, one, and two intermolecular disulfide bonds, respectively. In contrast, Norrin forms a homodimer in a head-to-tail fashion with a unique semicircular shape linked by three intermolecular disulfide bonds (Supplemental Fig. 3B). The importance of the Norrin dimer is supported by the functional consequences of mutations that disrupt the three disulfide bonds or the hydrophobic interactions at the dimer interface (Supplemental Fig. 4B,C).

\section{Mapping Fz4- and Lrp5/6-binding sites on the Norrin dimeric structure}

By mapping previous Norrin mutations (Smallwood et al. 2007) onto the current Norrin structure, we identified an exposed surface region, including amino acids R41, H43, V45, L61, Y120, and Y122, as the Fz4-binding site (Fig. 5A). Mutations in these residues significantly reduced $\mathrm{Fz} 4$ binding and Norrin signaling activity (Fig. 5B,C). As shown by sequence alignment (Supplemental Fig. 8A), all of these residues are conserved across species. The fact that a Norrin dimer contains two symmetric Fz4-binding sites suggests that the dimer can bind to two Fz4 CRDs, which was confirmed by immunoprecipitation (Fig. 2F). Two FEVR mutations were identified in the Fz4 CRD (M157V and M105V), which indicates that the base of the Fz4 CRD, including these two residues, is the surface for Norrin interaction (Xu et al. 2004).

In addition, we identified the Lrp5/6-binding site on the edge of Norrin, which comprises several positively charged residues (K54, R107, R109, and R115) and two hydrophobic residues (L52 and Y53) (Fig. 5A) and specifically binds to the BP1-2 domain (Fig. 5D). Mutation of these residues reduced signaling activity but did not affect Fz4 binding (Fig. 5C; Smallwood et al. 2007). The sequence alignment showed that all of the positively charged residues are completely conserved and that the hydrophobic residues L52 and Y53 are largely conserved (Supplemental Fig. 8A). The top concave surfaces of Lrp6 BP1 and BP2 contain a hydrophobic patch surrounded by negatively charged residues (Cheng et al. 2011). The hydrophobic and positively charged residues of Norrin may thus interact with the hydrophobic patch and the negatively charged residues on the top surface of BP1 or BP2.

\section{Assembly and activation of a Norrin-Fz4-Lrp5/6- Tspan12 signaling complex}

Our BRET and split-YFP data showed that Fz4 pre-exists as dimers on the cell membrane (Fig. 3A,D). Because Norrin also forms stable dimers, Norrin binds Fz4 with a 2:2 stoichiometry. We showed that Norrin binds to Lrp6
BP1-2 in addition to its binding to Fz4 (Fig. 4A). Previous work failed to detect an interaction between Norrin and Lrp5/6, which was likely due to the weak binding of Norrin to Lrp5/6 BP1-2 (affinity >40-fold lower than Norrin-Fz4 interactions). The Norrin homodimer with two symmetric edges can bind to one BP1-2 or two BP1-2 molecules (Fig. 7). Because a Norrin dimer can bind to both BP1 and BP2 domains of Lrp6 (Supplemental Fig. 6), we favor a model in which one Norrin dimer binds to one Lrp5/6 monomer, with each Norrin monomer binding to one BP domain of Lrp5/6 (Fig. 7). Synergistic binding of Norrin to both the BP1 and BP2 domains would further stabilize Norrin dimerization, consistent with the ability of Lrp5 and Lrp6 to partially rescue dimerizationcompromised Norrin mutants (Supplemental Fig. 4B,C), although we cannot exclude the possibility that one Norrin dimer can interact with two Lrp5/6 coreceptors.

Importantly, we found that a functional Lrp5/6 coreceptor is required for Norrin/Fz4 signaling (Fig. 6B) and that Norrin forms a ternary complex with both the Fz4 CRD and the Lrp6 ECD in vitro (Fig. 6A; Supplemental Fig. 7A). This suggests that the signaling mechanism for Norrin is likely very similar to that of canonical Wnts by inducing heterodimerization of Fz4 with Lrp5/6 (Cong et al. 2004). Furthermore, Fz4 activation by Norrin is enhanced by Tspan12, which specifically interacts with Fz4 (Fig. 6D) but not other Fz proteins such as Fz5 (Junge et al. 2009). We speculate that Tspan12 is a molecular chaperone that stabilizes Fz4 protein to facilitate Norrin interaction and signaling. Tspan 12 , by forming

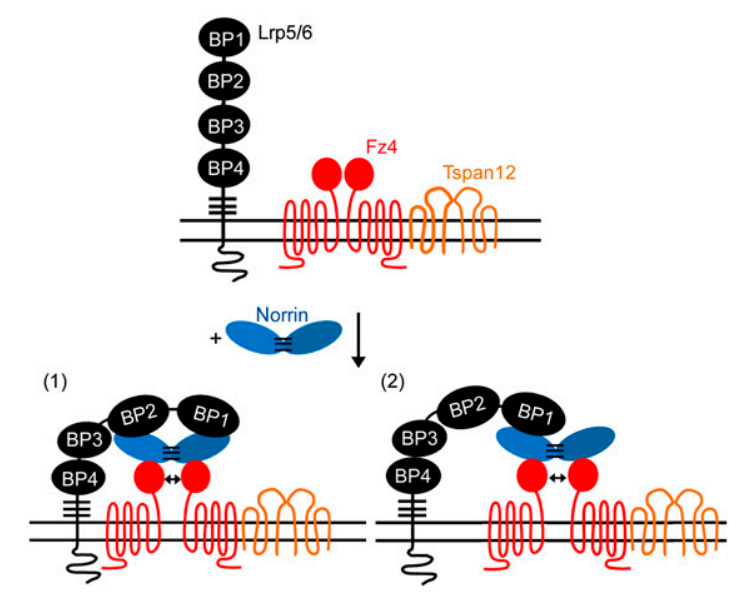

Figure 7. A model for Norrin-mediated Fz4 receptor activation. In the absence of Norrin, both Fz4 and Tspan12 form homodimers or oligomers. The interaction of $\mathrm{Fz} 4$ with Tspan 12 brings Fz4 into tetraspanin-enriched microdomains on the cell membrane. A Norrin dimer binds to an Fz4 dimer with high affinity. Simultaneously, Norrin also binds to Lrp5/6 BP1 and BP2 domains through its two edges (1) or, alternatively, predominantly to the Lrp5/6 BP1 domain (2). In the first binding mode, Norrin binds to one Lrp5/6 molecule, whereas in the second binding mode, Norrin can bind up to two Lrp5/6 molecules. The interaction of Norrin with $\mathrm{Fz} 4$ and Lrp5/6 brings their C-terminal tails closer for interaction and activation of the downstream $\beta$-catenin signaling pathway. 
heterodimers with Fz4, may compartmentalize Fz4 into tetraspanin-enriched microdomains on the cell membrane (Bailey et al. 2011) to facilitate Norrin/Fz4-mediated $\beta$-catenin signaling.

\section{Structural basis of Norrie disease mutations}

In spite of its small size, $>100$ disease-causing Norrin mutations have been identified in human patients (Ye et al. 2010). The Norrin structure provided us with an opportunity to examine the molecular basis of diseasecausing missense mutations (Supplemental Table 2; Supplemental Fig. 3C). Based on the structure, the majority of these mutations can be categorized into five different groups: cysteine residue mutations, dimer interface mutations, hydrophobic core mutations, Fz4-binding site mutations, and Lrp5/6-binding site mutations (Supplemental Table 2). The Norrin structure readily explains these disease-causing mutations, as they would compromise protein functions by affecting protein folding and stability, dimerization, or functional interaction with $\mathrm{Fz} 4$ and Lrp5/6. The large number of disease-causing mutations in Norrin suggests that this protein is very sensitive to perturbations, which is consistent with the multiple functions (protein folding, dimerization, and receptor and coreceptor binding) that it mediates.

\section{The Norrin structure may serve as a model for mucins and von Willebrand factor}

In a new sequence homology search, we found that Norrin is most closely related to the C-terminal domains of mucins and von Willebrand factor, two extracellular proteins with multiple domain structures. While only seven of the 11 cysteines in Norrin are conserved in TGF- $\beta$, all 11 cysteines are conserved in mucins and von Willebrand factor (Supplemental Fig. 8B). The structure of Norrin may therefore also represent that of mucins and von Willebrand factor C-terminal domains. In particular, the C55-C110 disulfide bond is conserved in Norrin, mucins, and von Willebrand factor (Supplemental Fig. $8 \mathrm{~B}$, blue stars), whereas TGF- $\beta$ proteins have a unique disulfide bond that is not found in those three proteins (Supplemental Fig. 8B, green stars). Moreover, the three-disulfide bond-linked dimer is a conserved feature of these proteins (Supplemental Fig. 8B, red stars), and mutational studies of pig submaxillary mucin also identified three cysteines that may be involved in forming the intermolecular disulfide bonds of the mucin dimer (PerezVilar and Hill 1998). Both mucins and von Willebrand factor can modulate Wnt signaling through interaction with other proteins (Rey and Ellies 2010). These domains in mucins and von Willebrand factor could function as dimerization and signaling domains, for which receptors remain to be identified.

In summary, we determined the crystal structure of Norrin, which revealed a stable dimer with three intermolecular disulfide bonds. Structure-based mapping revealed separate sites on the Norrin surface for binding of Fz4 and Lrp5/6, and Norrin forms a ternary complex with the Fz4 CRD and the Lrp6 ECD in vitro.
We propose that dimeric Norrin activates Fz4 by inducing heterodimerization of $\mathrm{Fz} 4$ with $\mathrm{Lrp} 5 / 6$ and that it may also induce conformational changes in the Fz4-Lrp5 signaling complex. These results provide important mechanistic insights into the assembly and activation of the Norrin-Fz4-Lrp5/6-Tspan12 signaling complex and unify the roles of $\operatorname{Lrp} 5 / 6$ as the common coreceptors for both Wnt and Norrin signaling.

\section{Materials and methods}

\section{DNA plasmids}

The TKRlu plasmid with the Rlu gene under the control of the thymidine kinase promoter was used as a transfection control (Promega). Human full-length Lrp5 and Lrp6 plasmids have been described (Holmen et al. 2005). The Lrp5-NT truncation construct was created by cloning the Lrp5 ECD, the TM domain, and eight residues following the TM domain (residues 1-1416) into the pcDNA6 vector with a v5 and a His6 tag at the $\mathrm{C}$ terminus. The Lrp6 BP1 (residues 20-325) and BP2 (residues 325-631) fragments were cloned into a pcDNA6 vector that had been previously cloned with a murine Igк leader sequence (pcDNA6IgL) plus Fc and His6 tags to create Lrp6BP1-FcH6 and Lrp6BP2FcH6 expression constructs. Human Norrin, Fz4, and Fz8 plasmids were from Open Biosystems. Xenopus Fz8 was from Addgene. The pHL-FcH6 vector from E. Yvonne Jones was cloned with a murine Igк leader sequence to create the pHL-IgL-FcH6 vector, which allows target protein secretion into medium supernatant. The CRD regions of Fz4 (residues 40-164) or Fz8 (residues 28-155) were cloned into the pHL-IgL-FcH6 vector to create the Fz4-FcH6 and Fz8-FcH6 fusion constructs. To purify the Fz4 CRD, a thrombin site was engineered between the Fz4 CRD and the Fc tag. The Fz4 CRD was also cloned in fusion with T4 lysozyme (residues 2-161, with C54T and C97A mutations) and included a v5 and His6 tandem tag at the $\mathrm{C}$ terminus (Fz4T4L-v5H6). T4 lysozyme with the same tandem tag was used as a negative control (T4L-v5H6). Both cDNAs were cloned into the pcDNA6-IgL vector. The full-length human Fz4 cDNA was first cloned into the pcDNA3.1 expression vector (Invitrogen). The v5, YFP, or Rlu cDNAs were subsequently cloned at the protein $\mathrm{C}$ terminus of $\mathrm{Fz} 4$ to create an in-frame fusion protein for v5-, Rlu-, and YFP-tagged Fz4 receptor constructs. The YFP-N-tagged (residues 1-158) and YFP-C-tagged (residues 159-239) Fz4 constructs were made by cloning YFP-N or YFP-C fragments at the protein C terminus of Fz4. Human Norrin (residues 25-133) in fusion with $\mathrm{MBP}$ at its $\mathrm{N}$ terminus was cloned into the first cloning site of the pETDuetl vector (Novagen), which also included a Dsbc cDNA at the second cloning site. For in vivo biotinylation of MBP-Norrin, a DNA oligonucleotide duplex encoding a biotinylation peptide (AviTag) was inserted in-frame at the $5^{\prime}$ end of the MBP-Norrin cDNA. In addition, the biotin ligase (BirA) gene with a T7 promoter was cloned downstream from MBP-Norrin cDNA. Coexpression of MBP-Norrin and BirA in the presence of biotin allowed in vivo biotinylation of MBP-Norrin (Smith et al. 1998). The MBP-Norrin cDNA was also cloned into pcDNA6-IgL vector. Norrin was cloned into the pHL-IgL vector for mammalian expression. All Norrin mutations were generated by PCR. Human Tspan12 (DNASU Plasmid Repository) was cloned into pCDNA3.1 expression vector (Invitrogen). The YFP or Rlu cDNAs were cloned at the C terminus of Tspan 12 to create an in-frame fusion for Rlu- and YFP-tagged Tspan12 constructs. All DNA constructs were verified by automated DNA sequencing. 


\section{Protein expression and purification}

Norrin was expressed as a fusion protein with MBP at its $\mathrm{N}$ terminus in the E. coli strain Origami B (DE3) (Novagen) as previously described (Pioszak and Xu 2008). The purification protocol was as previously described, with the following modifications: First, the fusion protein did not contain a His6 tag and was purified by amylose affinity chromatography via the MBP tag. Then MBP-Norrin protein was refolded in a solution of $1 \mathrm{M} \mathrm{L}$-arginine, $20 \mathrm{mM}$ Tris ( $\mathrm{pH} 8.5$ ), $0.5 \mathrm{M} \mathrm{NaCl}, 1 \mathrm{mM} \mathrm{GSH}$, $1 \mathrm{mM}$ GSSG, and $1 \mathrm{mM}$ EDTA. The active dimeric protein was further purified on a Heparin column (GE Healthcare) followed by a Superdex 120 gel filtration column (GE Healthcare). Protein concentrations were determined by the Bradford method (Bradford 1976). The MBP-tagged Norrin mutant proteins were purified with a protocol similar to that used for the wild type. The expression and purification of Lrp6 BP fragments have been described (Cheng et al. 2011). The Lrp6BP1-FcH6, Lrp6BP2FcH6, Fz4-Fc, Fz8-Fc, Fz4-T4L-v5H6, and T4L-v5H6 proteins were expressed by transient transfection of HEK293 cells with the corresponding DNA using Lipofectamine 2000 (Invitrogen). The medium supernatants were collected after $4 \mathrm{~d}$. To purify proteins from medium supernatant, samples were dialyzed against TBS buffer (20 mM Tris at pH 8.0, 0.15 M NaCl, 5\% glycerol) before loading onto a nickel chelating column (GE Healthcare). To purify the Fz4 CRD, purified Fz4-th-FcH6 protein was digested with thrombin (1:500) overnight at room temperature, and the FcH6 tag was separated from the Fz4 CRD by a nickel chelating column.

\section{Crystallization and structure determination}

The MBP-Norrin protein in $20 \mathrm{mM}$ Tris (pH 8.0), $0.1 \mathrm{M} \mathrm{NaCl}, 5 \%$ glycerol, $1 \mathrm{mM}$ maltose, and $1 \mathrm{mM}$ EDTA was concentrated to $\sim 5 \mathrm{mg} / \mathrm{mL}$ before we set up crystallization trials using the Phoenix crystallization robot. Several conditions yielded very good-looking crystals, but they only diffracted to $\sim 6 \AA$. We reasoned that the low resolution may be due to the flexible $\mathrm{N}$ terminus of Norrin. A truncation construct of Norrin (residues 31-133) fused with MBP at its $\mathrm{N}$ terminus was created. The encoding protein was expressed and purified with a protocol similar to that used for the wild-type protein. The function for this truncated protein was shown by binding assays to be very similar to that of the wild-type protein (data not shown). After screening many crystals at the APS synchrotron, we obtained crystal diffraction data to $2.4 \AA$ from crystals grown in $15 \%$ PEG $3350,0.1 \mathrm{M}$ sodium acetate $(\mathrm{pH} 4.6)$, and $0.2 \mathrm{M}$ ammonium acetate. The crystal structure of the MBP-Norrin protein was determined by molecular replacement using MBP as an initial model. Attempts to use the structure of TGF- $\beta 3$, a remote homolog of Norrin, as a search model failed to yield a correct solution. The model for Norrin was therefore built de novo with phases established by the MBP structure. The Coot program was used for model building (Emsley and Cowtan 2004). The electron density for Norrin was gradually improved over many cycles of manual model building and refinements. The final model of Norrin included all of the residues, and the electron density for most of the residues (including side chains) can be clearly seen except for that of a loop region (residues 111-116). The diffraction data and refinement statistics are listed in Table 1. The MBP-Norrin structure was deposited into Protein Data Bank (PDB) with PDB code 4MY2.

\section{AlphaScreen binding assay}

The AlphaScreen assay is a bead-based luminescence proximity assay. The binding between biotinylated MBP-Norrin and His6- tagged Fz4-Fc or Fz8-Fc or His8-tagged Lrp6 ECD proteins was determined by the AlphaScreen assay using a hexahistidine detection kit from PerkinElmer. In this assay, biotinylated MBPNorrin was attached to streptavidin-coated donor beads, and His6tagged protein was attached to nickel chelate-coated acceptor beads. When the donor and acceptor beads were brought into proximity by the interaction between MBP-Norrin and His6tagged protein, illuminating the sample with a laser at $680 \mathrm{~nm}$ released singlet oxygen molecules from donor beads to acceptor beads, which then elicited a strong emission of light at a shorter wavelength $(520 \sim 620 \mathrm{~nm})$. The binding mixtures, containing the indicated amounts of proteins, $10-20 \mu \mathrm{g} / \mathrm{mL}$ streptavidincoated "donor" beads, and nickel chelate-coated "acceptor" beads, were incubated in $50 \mathrm{mM}$ MOPS (pH 7.4), $100 \mathrm{mM} \mathrm{NaCl}$, and $0.1 \mathrm{mg} / \mathrm{mL}$ BSA for $2-5 \mathrm{~h}$ followed by data collection using an Envision plate reader (PerkinElmer). For competition assays, increasing concentrations of unlabeled protein were added to the two labeled proteins.

\section{Biolayer interference assays}

Binding curves were measured by biolayer interferometry using an Octet Red instrument (ForteBio). For the biolayer interferometry assay, a layer of molecules attached to the tip of the biosensor created an interference pattern at the detector. Any change in the number of molecules bound due to protein-protein interactions caused a measured shift in the interferometric profile. When this shift was measured over a period of time and its magnitude was plotted as a function of time, a classic association/dissociation curve was obtained. The protein A sensor (immobilized with protein $\mathrm{A}$, a bacterial surface protein that binds the Fc region of the heavy chain of most antibodies) or anti-human IgG Fc capture sensor (immobilized with anti-human Fc-specific antibody) was used to bind Fz4-FcH6, Fz8-FcH6, Lrp6 BP2-FcH6, or human IgG proteins in kinetics buffer (PBS at pH 7.4, 0.01\% BSA, $0.002 \%$ Tween 20) or as noted otherwise. The protein-loaded biosensors were incubated sequentially with kinetics buffer in the baseline step, the purified MBP-Norrin in solution in the association step, and kinetics buffer in the dissociation step to detect their binding to MBP-Norrin protein.

\section{Cell-based luciferase assay}

293STF cells with an integrated "super-top-flash" TCF-luciferase reporter were maintained in DMEM (Gibco) with $5 \%$ fetal bovine serum (FBS). Cells were plated in a 24 -well plate $24 \mathrm{~h}$ prior to transfection. Cells were transfected with the indicated plasmids plus 5-10 ng of the control plasmid TKRlu (constitutive expression of Rlu) using Lipofectamine 2000 (Invitrogen). The medium was changed to the low-serum opti-MEM (Invitrogen) on day 2. For experiments involving Norrin DNA transfections, cells were incubated for another $24 \mathrm{~h}$ before harvesting. For experiments involving protein treatments, MBP-Norrin protein was added at the time of medium change, and cells were incubated with the indicated protein for $24 \mathrm{~h}$ before harvesting. The cells were lysed with 100-150 $\mu \mathrm{L}$ of passive lysis buffer (Promega) for $15 \mathrm{~min}$ at room temperature, and the firefly luciferase and Rlu activities were measured on an Envision luminometer (PerkinElmer) with the Dual-Luciferase assay kit (Promega). Firefly luciferase raw data were normalized to Rlu raw data.

\section{Immunoprecipitation}

To produce secreted Fz4-T4L-v5H6 or T4L-v5H6 proteins, HEK293 cells in a 10-cm plate were transfected with $12 \mu \mathrm{g}$ of the Fz4-T4L-v5H6 or T4L-v5H6 DNAs by Lipofectamine 2000 
(Invitrogen), and medium supernatants were collected after $4 \mathrm{~d}$ of transfection. To produce Lrp5NT-v5H6 protein, HEK293 cells in a $10-\mathrm{cm}$ plate were transfected with $12 \mu \mathrm{g}$ of Lrp5NT-v5H6 DNA for $2 \mathrm{~d}$. After that, cells were harvested with Cell Dissociation solution (Sigma) and lysed in $300 \mu \mathrm{L}$ of cell lysis buffer (Cell Signaling) for $30 \mathrm{~min}$ on ice. Cell debris was removed by centrifugation. For immunoprecipitation, $30 \mu \mathrm{L}$ of v5-agarose beads (Sigma) was used to pull down $\sim 2 \mu \mathrm{g}$ of Fz4-T4L-v5H6 or T4Lv5H6 from medium supernatant or Lrp5NT-v5H6 protein from $100 \mu \mathrm{L}$ of cell lysate. The v5 beads were then incubated with or without $10 \mu \mathrm{g}$ of MBP-Norrin and $10 \mu \mathrm{g}$ of Fz4FcH6 proteins for $1 \mathrm{~h}$ at $4^{\circ} \mathrm{C}$ with mixing. The v5 beads were washed with $1 \times$ TBST buffer twice and then incubated with $100 \mu \mathrm{L}$ of $2 \times$ SDS loading buffer to release the bound proteins. The samples were analyzed by Western blot as described previously (Ke et al. 2009, 2012). The blot was probed first with anti-hum IgG horseradish peroxidase (HRP) (Santa Cruz Biotechnology) for Fc4-FcH6 protein. The blot was then stripped and reprobed with a goat anti-Norrin antibody (R\&D Systems). The blot was stripped again and reprobed with a mouse anti-v5 antibody (Thermo Scientific).

\section{BRET assays for receptor oligomerization}

COS- 1 cells were seeded at a density of $0.5 \times 10^{6}$ cells per dish in $10-\mathrm{cm}$ tissue culture dishes in DMEM supplemented with 5\% FetalClone II. When the cells reached $80 \%$ confluence, they were transfected with $3 \mu \mathrm{g}$ of DNA per dish using the diethylaminoethyl (DEAE)-dextran method (Harikumar et al. 2007). Assays were performed 48-72 h later. BRET studies were performed using cells in suspension and a 2103 Envision plate reader (PerkinElmer) configured with a $<700-\mathrm{nm}$ dichroic mirror and dual emission filter sets for luminescence (460 nm; bandwidth, $25 \mathrm{~nm}$ ) and fluorescence (535 nm; bandwidth, $25 \mathrm{~nm}$ ). These studies used $\sim 25,000$ cells per well in 96-well Optiplates. The studies were initiated by adding $5 \mu \mathrm{M}$ coelenterazine $h$, a specific substrate for Rlu, after which the luminescence and fluorescence signals were promptly collected. Total YFP fluorescence emission was also acquired to determine acceptor concentration by exciting the samples at $485 \mathrm{~nm}$ and detecting the emission at $525 \mathrm{~nm}$. The net BRET ratios were calculated based on the ratio of emission signals from YFP and Rlu, and corrected BRET ratios were calculated as described previously (Harikumar et al. 2007). Saturation BRET studies were performed to evaluate the specificity of the BRET signals as described previously (Harikumar et al. 2007).

\section{Fluorescence microscopy and fluorescence spectroscopy}

Fluorescence microscopy was used to demonstrate YFP fluorescence at the surface of the transfected cells. COS-1 cells were transfected with either the intact YFP construct or the complementary YFP-N and YFP-C constructs, as described previously (Harikumar et al. 2008a,b). Cell surface YFP fluorescence was evaluated using a Zeiss Axiovert 200M epifluorescence inverted microscope with a dedicated YFP filter set (excitation, $480 \mathrm{~nm}$; dichroic mirror, Q515 lp; emission, $525 \mathrm{~nm}$ ). Images were collected using a monochromatic ORCA-12ER CCD camera (Hamamatsu) with QED-InVivo 2.039 acquisition software (Media Cybernetics). Steady-state fluorescence intensity measurements were performed in a FluoroMax-3 fluorometer (SPEX Industries) with samples at room temperature in a $1-\mathrm{mL}$ quartz cuvette. Transfected cells were harvested and transferred into a $1-\mathrm{mL}$ cuvette where the sample was excited using $480 \mathrm{~nm}$ light, and YFP fluorescence emission was monitored between $500 \mathrm{~nm}$ and $600 \mathrm{~nm}$. Background fluorescence was corrected by analogous measurements using untransfected cells. Fluorescence anisotropy measurements were performed as described previously (Harikumar et al. 2008a).

\section{Triton $X-114$ phase separation assay}

The phase separation assay was performed as previously described (Willert et al. 2003). HEK293 cells were transiently transfected with MBP-Norrin expression vector, and the medium supernatant was collected after 4 d and concentrated. As a control, HEK293 cells were transiently transfected with MBP-Rhodopsin expression vector for $1 \mathrm{~d}$, and the cells were lysed using a solution of $0.5 \%$ n-dodecyl- $\beta$-D-maltoside, $0.1 \%$ cholesterol, $150 \mathrm{mM} \mathrm{NaCl}$, and $20 \mathrm{mM}$ Tris $(\mathrm{pH} 7.5)$ at $4{ }^{\circ} \mathrm{C}$. The cell lysate was centrifuged at $40,000 \mathrm{rpm}$ for $30 \mathrm{~min}$, and the supernatant was used for the phase separation assay. MBP-Norrin conditioned medium or MBPRhodopsin lysate were mixed 1:1 with ice-cold $4.5 \%$ Triton $\mathrm{X}-114,150 \mathrm{mM} \mathrm{NaCl}$, and $10 \mathrm{mM}$ Tris- $\mathrm{HCl}$ (pH 7.5); incubated for $5 \mathrm{~min}$ on ice and then $5 \mathrm{~min}$ at $31^{\circ} \mathrm{C}$; and centrifuged at $2000 \mathrm{~g}$ for $5 \mathrm{~min}$ at $31^{\circ} \mathrm{C}$. The top, aqueous phase was separated from the bottom Triton X-114 detergent phase, and equal volumes were analyzed for Western blot.

\section{Acknowledgments}

We thank Dr. Joseph S. Brunzelle for assistance with data collection, Drs. David Tolbert and Kuntal Pal for help during structure determination, Alicja Ball and Mary-Lou Augustine for assistance with cell culture for the BRET studies, and Heather Schumacher for assistance with flow cytometry. We also thank Dr. Catherine Berlot (Geisinger Clinic, Danville, PA) for the N-terminal (1-158) and C-terminal (159-239) fragments of YFP. In addition, we thank David E. Nadziejka for technical editing assistance in preparing the manuscript. This work was supported in part by National Institute of Health grants DK71662 (to H.E.X.) and DK46577 (to L.J.M.).

\section{References}

Bailey RL, Herbert JM, Khan K, Heath VL, Bicknell R, Tomlinson MG. 2011. The emerging role of tetraspanin microdomains on endothelial cells. Biochem Soc Trans 39: 1667-1673.

Berger W. 1998. Molecular dissection of Norrie disease. Acta Anat (Basel) 162: 95-100.

Bourhis E, Wang W, Tam C, Hwang J, Zhang Y, Spittler D, Huang OW, Gong Y, Estevez A, Zilberleyb I, et al. 2011. Wnt antagonists bind through a short peptide to the first $\beta$-propeller domain of LRP5/6. Structure 19: 1433-1442.

Bradford MM. 1976. A rapid and sensitive method for the quantitation of microgram quantities of protein utilizing the principle of protein-dye binding. Anal Biochem 72: 248-254.

Chen ZY, Battinelli EM, Fielder A, Bundey S, Sims K, Breakefield XO, Craig IW. 1993. A mutation in the Norrie disease gene (NDP) associated with X-linked familial exudative vitreoretinopathy. Nat Genet 5: 180-183.

Cheng Y, Prusoff WH. 1973. Relationship between the inhibition constant (K1) and the concentration of inhibitor which causes $50 \%$ inhibition (I50) of an enzymatic reaction. Biochem Pharmacol 22: 3099-3108.

Cheng Z, Biechele T, Wei Z, Morrone S, Moon RT, Wang L, Xu W. 2011. Crystal structures of the extracellular domain of LRP6 and its complex with DKK1. Nat Struct Mol Biol 18: 1204-1210.

Cong F, Schweizer L, Varmus H. 2004. Wnt signals across the plasma membrane to activate the $\beta$-catenin pathway by forming oligomers containing its receptors, Frizzled and LRP. Development 131: 5103-5115. 
Emsley P, Cowtan K. 2004. Coot: Model-building tools for molecular graphics. Acta Crystallogr D Biol Crystallogr 60: 2126-2132.

Gong Y, Slee RB, Fukai N, Rawadi G, Roman-Roman S, Reginato AM, Wang H, Cundy T, Glorieux FH, Lev D, et al. 2001. LDL receptor-related protein 5 (LRP5) affects bone accrual and eye development. Cell 107: 513-523.

Harikumar KG, Pinon DI, Miller LJ. 2007. Transmembrane segment IV contributes a functionally important interface for oligomerization of the Class II G protein-coupled secretin receptor. J Biol Chem 282: 30363-30372.

Harikumar KG, Happs RM, Miller LJ. 2008a. Dimerization in the absence of higher-order oligomerization of the G proteincoupled secretin receptor. Biochim Biophys Acta 1778: 25552563.

Harikumar KG, Morfis MM, Sexton PM, Miller LJ. 2008b. Pattern of intra-family hetero-oligomerization involving the G-protein-coupled secretin receptor. J Mol Neurosci 36: 279285.

He X, Semenov M, Tamai K, Zeng X. 2004. LDL receptor-related proteins 5 and 6 in Wnt $/ \beta$-catenin signaling: Arrows point the way. Development 131: 1663-1677.

Holmen SL, Robertson SA, Zylstra CR, Williams BO. 2005. Wnt-independent activation of $\beta$-catenin mediated by a Dkk1-Fz5 fusion protein. Biochem Biophys Res Commun 328: $533-539$.

Huelsken J, Behrens J. 2002. The Wnt signalling pathway. J Cell Sci 115: 3977-3978.

Janda CY, Waghray D, Levin AM, Thomas C, Garcia KC. 2012. Structural basis of Wnt recognition by Frizzled. Science 337: 59-64.

Junge HJ, Yang S, Burton JB, Paes K, Shu X, French DM, Costa M, Rice DS, Ye W. 2009. TSPAN12 regulates retinal vascular development by promoting Norrin- but not Wnt-induced FZD4/ $\beta$-catenin signaling. Cell 139: 299-311.

Kaykas A, Yang-Snyder J, Heroux M, Shah KV, Bouvier M, Moon RT. 2004. Mutant Frizzled 4 associated with vitreoretinopathy traps wild-type Frizzled in the endoplasmic reticulum by oligomerization. Nat Cell Biol 6: 52-58.

Ke I, Chelvarajan RL, Sindhava V, Robertson DA, Lekakis L, Jennings CD, Bondada S. 2009. Anomalous constitutive Src kinase activity promotes B lymphoma survival and growth. Mol Cancer 8: 132.

Ke J, Zhang C, Harikumar KG, Zylstra-Diegel CR, Wang L, Mowry LE, Miller LJ, Williams BO, Xu HE. 2012. Modulation of $\beta$-catenin signaling by glucagon receptor activation. PLOS ONE 7: e33676.

Logan CY, Nusse R. 2004. The Wnt signaling pathway in development and disease. Annu Rev Cell Dev Biol 20: 781-810.

Luhmann UF, Meunier D, Shi W, Luttges A, Pfarrer C, Fundele R, Berger W. 2005. Fetal loss in homozygous mutant Norrie disease mice: A new role of Norrin in reproduction. Genesis 42: $253-262$.

Nusse R. 2003. Wnts and Hedgehogs: Lipid-modified proteins and similarities in signaling mechanisms at the cell surface. Development 130: 5297-5305.

Ohlmann A, Seitz R, Braunger B, Seitz D, Bosl MR, Tamm ER. 2010. Norrin promotes vascular regrowth after oxygen-induced retinal vessel loss and suppresses retinopathy in mice. I Neurosci 30: 183-193.

Perez-Vilar J, Hill RL. 1997. Norrie disease protein (norrin) forms disulfide-linked oligomers associated with the extracellular matrix. J Biol Chem 272: 33410-33415.

Perez-Vilar J, Hill RL. 1998. The carboxyl-terminal 90 residues of porcine submaxillary mucin are sufficient for forming disulfide-bonded dimers. J Biol Chem 273: 6982-6988.
Pioszak AA, Xu HE. 2008. Molecular recognition of parathyroid hormone by its G protein-coupled receptor. Proc Natl Acad Sci 105: 5034-5039.

Pioszak AA, Parker NR, Suino-Powell K, Xu HE. 2008. Molecular recognition of corticotropin-releasing factor by its G-protein-coupled receptor CRFR1. J Biol Chem 283: 3290032912.

Rehm HL, Zhang DS, Brown MC, Burgess B, Halpin C, Berger W, Morton CC, Corey DP, Chen ZY. 2002. Vascular defects and sensorineural deafness in a mouse model of Norrie disease. J Neurosci 22: 4286-4292.

Rey JP, Ellies DL. 2010. Wnt modulators in the biotech pipeline. Dev Dyn 239: 102-114.

Seitz R, Hackl S, Seibuchner T, Tamm ER, Ohlmann A. 2010. Norrin mediates neuroprotective effects on retinal ganglion cells via activation of the $\mathrm{Wnt} / \beta$-catenin signaling pathway and the induction of neuroprotective growth factors in Muller cells. J Neurosci 30: 5998-6010.

Shastry BS, Trese MT. 2003. Overproduction and partial purification of the Norrie disease gene product, norrin, from a recombinant baculovirus. Biochem Biophys Res Commun 312: 229-234.

Smallwood PM, Williams J, Xu Q, Leahy DJ, Nathans J. 2007. Mutational analysis of Norrin-Frizzled4 recognition. I Biol Chem 282: 4057-4068.

Smith PA, Tripp BC, DiBlasio-Smith EA, Lu Z, LaVallie ER, McCoy JM. 1998. A plasmid expression system for quantitative in vivo biotinylation of thioredoxin fusion proteins in Escherichia coli. Nucleic Acids Res 26: 1414-1420.

Sun PD, Davies DR. 1995. The cystine-knot growth-factor superfamily. Annu Rev Biophys Biomol Struct 24: 269-291.

Takada R, Satomi Y, Kurata T, Ueno N, Norioka S, Kondoh H, Takao T, Takada S. 2006. Monounsaturated fatty acid modification of Wnt protein: Its role in Wnt secretion. Dev Cell 11: 791-801.

Tamai K, Semenov M, Kato Y, Spokony R, Liu C, Katsuyama Y, Hess F, Saint-Jeannet JP, He X. 2000. LDL-receptor-related proteins in Wnt signal transduction. Nature 407: 530-535.

Wang Y, Rattner A, Zhou Y, Williams I, Smallwood PM, Nathans J. 2012. Norrin/Frizzled4 signaling in retinal vascular development and blood brain barrier plasticity. Cell 151: 1332-1344.

Wang C, Wu H, Katritch V, Han GW, Huang XP, Liu W, Siu FY, Roth BL, Cherezov V, Stevens RC. 2013. Structure of the human smoothened receptor bound to an antitumour agent. Nature 497: 338-343.

Willert K, Brown JD, Danenberg E, Duncan AW, Weissman IL, Reya T, Yates JR 3rd, Nusse R. 2003. Wnt proteins are lipidmodified and can act as stem cell growth factors. Nature 423: 448-452.

Xu Q, Wang Y, Dabdoub A, Smallwood PM, Williams J, Woods C, Kelley MW, Jiang L, Tasman W, Zhang K, et al. 2004. Vascular development in the retina and inner ear: Control by Norrin and Frizzled-4, a high-affinity ligand-receptor pair. Cell 116: 883-895.

Ye X, Wang Y, Nathans J. 2010. The Norrin/Frizzled4 signaling pathway in retinal vascular development and disease. Trends Mol Med 16: 417-425. 


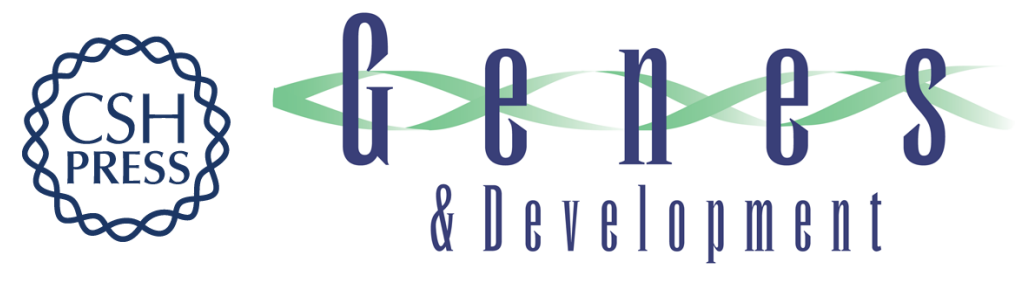

\title{
Structure and function of Norrin in assembly and activation of a Frizzled 4-Lrp5/6 complex
}

\author{
Jiyuan Ke, Kaleeckal G. Harikumar, Clara Erice, et al.
}

Genes Dev. 2013, 27:

Access the most recent version at doi:10.1101/gad.228544.113

\section{Supplemental http://genesdev.cshlp.org/content/suppl/2013/11/01/27.21.2305.DC1 Material}

References This article cites 43 articles, 15 of which can be accessed free at: http://genesdev.cshlp.org/content/27/21/2305.full.html\#ref-list-1

Creative This article is distributed exclusively by Cold Spring Harbor Laboratory Press for the first Commons six months after the full-issue publication date (see

License http://genesdev.cshlp.org/site/misc/terms.xhtml). After six months, it is available under a Creative Commons License (Attribution-NonCommercial 3.0 Unported), as described at http://creativecommons.org/licenses/by-nc/3.0/.

Email Alerting Receive free email alerts when new articles cite this article - sign up in the box at the top Service right corner of the article or click here.

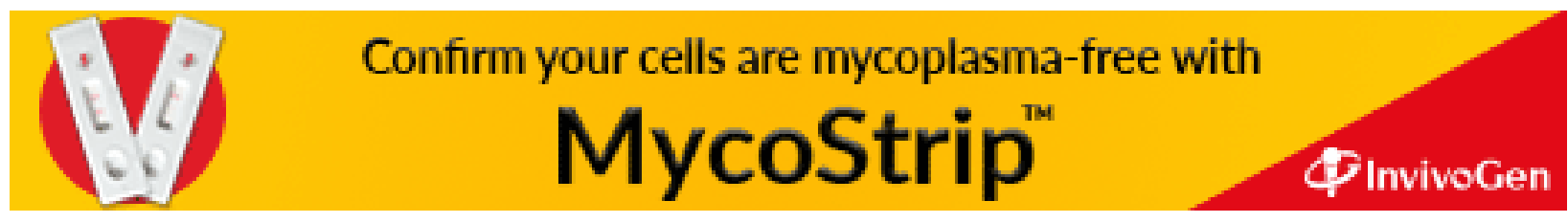

